\title{
The systemic nature of CKD
}

\section{Carmine Zoccali ${ }^{1}$, Raymond Vanholder ${ }^{2}$, Ziad A. Massy ${ }^{3-7}$, Alberto Ortiz ${ }^{8}$, Pantelis Sarafidis ${ }^{9}$, Friedo W. Dekker ${ }^{10}$, Danilo Fliser ${ }^{11}$, Denis Fouque ${ }^{12}$, Gunnar H. Heine ${ }^{11}$, Kitty J. Jager ${ }^{13}$, Mehmet Kanbay ${ }^{14}$, Francesca Mallamaci ${ }^{1,15}$, Gianfranco Parati' ${ }^{16}$, Patrick Rossignol ${ }^{7,17-20}$, Andrzej Wiecek ${ }^{21}$ and Gerard London ${ }^{22}$; on behalf of the European Renal and Cardiovascular Medicine (EURECA-m) Working Group of the European Renal Association - European Dialysis Transplantation Association (ERA-EDTA)}

Abstract | The accurate definition and staging of chronic kidney disease (CKD) is one of the major achievements of modern nephrology. Intensive research is now being undertaken to unravel the risk factors and pathophysiologic underpinnings of this disease. In particular, the relationships between the kidney and other organs have been comprehensively investigated in experimental and clinical studies in the last two decades. Owing to technological and analytical limitations, these links have been studied with a reductionist approach focusing on two organs at a time, such as the heart and the kidney or the bone and the kidney. Here, we discuss studies that highlight the complex and systemic nature of CKD. Energy balance, innate immunity and neuroendocrine signalling are highly integrated biological phenomena. The diseased kidney disrupts such integration and generates a high-risk phenotype with a clinical profile encompassing inflammation, protein-energy wasting, altered function of the autonomic and central nervous systems and cardiopulmonary, vascular and bone diseases. A systems biology approach to CKD using omics techniques will hopefully enable in-depth study of the pathophysiology of this systemic disease, and has the potential to unravel critical pathways that can be targeted for CKD prevention and therapy.

The definition, classification and evaluation of chronic kidney disease (CKD) is a major achievement of modern clinical nephrology ${ }^{1}$. The widely accepted description of the disease and the staging system based on measurements of kidney function that can be reliably applied on a vast scale at reasonable cost, has been a formidable stimulus for clinical research ${ }^{2}$. This standardized way of looking at renal diseases has provided a common vocabulary and a shared approach to these diseases in clinical practice ${ }^{2,3}$. The natural history of the complications of CKD is, however, still being established in detail, and the prevalence and severity of the main metabolic and endocrine alterations associated with precise stages of $\mathrm{CKD}$ are currently being investigated ${ }^{4}$.

CKD has repercussions for other organs. For example, reduced renal function leads to hypertension, which when combined with other conditions can cause left ventricular hypertrophy and dysfunction, and eventually heart failure ${ }^{5}$. The term crosstalk has become fashionable to describe the relationships between the kidney and other organs. On close scrutiny, however, crosstalk is loosely used in clinical and biological contexts that might not be linked to one another. For example, in cross-sectional studies, the presence of renal biomarkers or histopathological changes in the kidney ${ }^{6}$ has been associated with the presence of endocrine factors or markers of distant organ dysfunction or damage (such as troponin ${ }^{7}, \mathrm{~B}$ natriuretic peptides ${ }^{8}$ or other cardiac biomarkers ${ }^{9}$ ). In most clinical studies, crosstalk is used to highlight bivariate associations such as bone-kidney crosstalk ${ }^{10}$, rather than to describe complex multiorgan relationships. The lax use of this term has facilitated the emergence of new syndromic constructs such as cardiorenal syndrome and CKDmineral and bone disorder (CKD-MBD), which has now gained the status of a syndrome ${ }^{11}$. In addition, the studies that described kidney-heart or kidney-bone relationships have questionable methodological underpinnings and largely fail to meet formal criteria for the definition of syndromes ${ }^{12,13}$.

The human body is a single biological entity. A reductionist approach can be valuable for the initial evaluation of phenomena that seem to be linked ${ }^{14}$, but reductionist constructs that evolve into criteria to identify new 


\section{Key points}

- CKD is an inherently systemic disease

- Energy balance, innate immunity and neuroendocrine control of organ function are highly integrated biological processes; such integration is disrupted by loss of kidney function, which generates a high risk clinical phenotype

- The clinical profile of chronic kidney disease (CKD) includes inflammation, malnutrition, altered activity of the autonomic and central nervous systems, and cardiopulmonary, vascular and bone disease

- The gut and the lung are emerging as critical mediators of the interaction between the kidney and the environment, and are involved in cardiovascular disease and other systemic complications of CKD

- Alterations in macrovascular and microvascular function induced by sleep apnoea, inflammation and oxidative stress increase the risk of brain disease in CKD

- The application of omics sciences will enable in-depth studies of the pathophysiology and treatment of CKD century ${ }^{17}$ and the detrimental systemic consequences of renal function loss were described in thorough detail 45 years ago $^{18}$.

In this Review, we describe the profound effect of renal function loss on several systems: energy metabolism, inflammation, neuroendocrine signalling, bone homeostasis, and the gastrointestinal, pulmonary and nervous systems. We provide examples of high-level integration of organ function that include the kidney and discuss how such integration is disrupted in CKD.

\section{CKD disrupts the energy-immunity link}

The regulation of energy balance is a fundamental homeostatic function of the human body that is orchestrated by diverse neuroendocrine pathways ${ }^{19}$. This function evolved, in part, to provide adequate energy levels to sustain short-lived inflammatory episodes, such as responses to infections or immune surveillance at the tissue level. Hence, immunity and metabolism are functionally coupled. The link between energy regulation and the immune system is also evident in daily life processes, such as the postprandial cytokine response to meal consumption ${ }^{20}$ or the effects of caloric and fat restriction on total leukocyte count ${ }^{21}$, and such coupling syndromes are problematic. Focusing on the relationship between the kidney and one or, at most, two distant organs ${ }^{15}$ does not take the unity of organisms into account and eclipses the systemic nature of chronic diseases ${ }^{16}$. The systemic complications of renal diseases were recognized at the beginning of the nineteenth
Author addresses

${ }^{1}$ CNR-IFC Clinical Epidemiology and Pathophysiology of Renal Diseases and Hypertension Unit, Ospedali Riuniti 89124 Reggio Calabria, Italy.

${ }^{2}$ Ghent University Hospital, Department of Nephrology, Department of Internal Medicine, University Hospital Gent, De Pintelaan 185, B9000 Ghent, Belgium.

${ }^{3}$ Division of Nephrology, Ambroise Paré Hospital, Assistance Publique Hôpitaux de Paris, 9 Avenue Charles de Gaulle, 92100 Boulogne-Billancourt, Paris.

${ }^{4}$ University of Paris Ouest-Versailles-Saint-Quentin-en-Yvelines (UVSQ), 55 Avenue de Paris, 78000 Versailles, France.

Inserm U-1018, Centre de recherche en épidémiologie et santé des populations (CESP), Equipe 5, Hôpital

Paul-Brousse,16 avenue Paul Vaillant-Couturier, 94807 Villejuif Cedex, France.

${ }^{6}$ Paris-Sud University (PSU), 15 Rue Georges Clemenceau, 91400 Orsay, France.

${ }^{7}$ French-Clinical Research Infrastructure Network (F-CRIN), Pavillon Leriche 2è étage CHU de Toulouse, Place Dr Baylac TSA40031, 31059 TOULOUSE Cedex 3, France.

${ }^{8}$ Fundación Jiménez Díaz, Universidad Autónoma de Madrid, Fundación Renal Iñigo Alvarez de Toledo, Madrid, Av. Reyes Católicos, 2, 28040 Madrid, Spain.

${ }^{9}$ Department of Nephrology, Hippokration Hospital, Thessaloniki, Konstantinoupoleos 49, Thessaloniki 546 42, Greece.

${ }^{10}$ Department of Clinical Epidemiology, Leiden University Medical Center, Albinusdreef 2, 2333 ZA Leiden, The

Netherlands.

${ }^{11}$ Department Internal Medicine IV- Renal and Hypertensive Disease -Saarland University Medical Centre Kirrberger Straß 66421 Homburg, Saar, Germany.

${ }^{12}$ Université de Lyon, UCBL, Carmen, Department of Nephrology, Centre Hospitalier Lyon-Sud, F-69495 Pierre Bénite, France.

${ }^{13}$ European Renal Association-European Dialysis and Transplant Association (ERA-EDTA) Registry, Department of Medical Informatics, Meibergdreef 9, 1105 AZ Amsterdam-Zuidoost, The Netherlands.

${ }^{14}$ Division of Nephrology, Department of Medicine,Koç University, Rumelifeneri Yolu 34450 Sarıyer Istanbul, Turkey.

${ }^{15}$ Nephrology, Dialysis and Transplantation Unit Ospedali Riuniti, 89124 Reggio Calabria Italy.

${ }^{16}$ Department of Cardiovascular, Neural and Metabolic Sciences, S. Luca Hospital, Istituto Auxologico Italiano \&

Department of Medicine and Surgery, University of Milan-Bicocca, Piazzale Brescia 20, Milan 20149, Italy.

${ }^{17}$ Inserm, Centre d'Investigations Cliniques-Plurithématique 1433, Cardiovascular and Renal Clinical Trialists (INI-CRCT),

Institut Lorrain du Cœur et des Vaisseaux Louis Mathieu, 4 rue Morvan, 54500 Vandoeuvre-les-Nancy, France

${ }^{18}$ Inserm U1116, Faculté de Médecine, Bâtiment D 1er étage, 9 avenue de la forêt de Haye - BP 184, 54500 Vandœuvrelès-Nancy Cedex, France

${ }^{19} \mathrm{CHU}$ Nancy, Département de Cardiologie, Institut Lorrain du Cœur et des Vaisseaux, 5 Rue du Morvan, 54500

Vandœuvre-lès-Nancy, France;

${ }^{20}$ Université de Lorraine, 34 Cours Léopold, 54000 Nancy, France.

${ }^{21}$ Department of Nephrology, Transplantation and Internal Medicine, Medical University of Silesia, Francuska 20/24

Street, Pl-40-027 Katowice, Poland.

${ }^{22}$ INSERM U970, Hopital Européen Georges Pompidou,20 Rue Leblanc, 75015 Paris, France. 


\section{Box $1 \mid$ Circadian control of the immune and neuroendocrine systems}

In the morning, activation of the hypothalamic-pituitary axis, the sympathetic system, the adrenal medulla and glucagon induces lipolysis, reduces lipogenesis, and triggers $\beta$-oxidation of fatty acids, glycogenolysis and gluconeogenesis ${ }^{32}$. Mobilization of these energy-rich fuels sustains daily activities and adjustments of the inflammatory-immune system such as leucocyte redistribution ${ }^{271}$ and polyclonal antibody production ${ }^{272}$. At midnight, the functioning level of these systems is at the lowest point, whereas melatonin ${ }^{273}$, growth hormone ${ }^{274}$ and prolactin ${ }^{275}$ are secreted after the initiation of the sleeping phase. This hormonal surge activates the immune system and determines the expansion of antigen-specific lymphocyte clones, antibody production ${ }^{276}$ and cytokine release ${ }^{277}$. On waking, the activity of the hypothalamic-pituitary and sympathetic systems is again at the zenith. At the same time circulating cytokines, which stimulate sympathetic activity ${ }^{278}$, reach a peak level ${ }^{279}$ and the cycle is restarted.

has an approximate circadian periodicity (BOX 1; FIG. 1). Metabolic alterations (such as diabetes and bone mineral disorders (hyperparathyroidism) and immune dysfunction (autoimmune diseases such as lupus) profoundly affect the kidney and can even cause renal function loss. Vice versa, renal dysfunction can affect metabolic and immune balances. Examples of the influence of the kidney on metabolism include the increase in frequency of insulin resistance that is associated with deterioration of renal function, and the high levels of innate immunity biomarkers, such as C-reactive protein (CRP) and IL-6, that are present in patients with advanced stages of CKD.

\section{Energy storage and immunity}

Energy-rich fuels are required to mount and sustain short-term inflammatory responses to environmental threats ${ }^{19}$. Glucose, glutamine and other glucogenic amino acids, free fatty acids and ketones are indispensable for the adequate functioning of the immune system. Cytokines such as IL-7 and IL-4, and hormones such as insulin and leptin, stimulate the uptake of glucose in immune cells via upregulation of glucose transport$\mathrm{ers}^{22,23}$. When the immune system is activated, muscle proteins are degraded to supply amino acids for gluconeogenesis, a process that is fundamental to guarantee adequate provision of glucose to the brain.

Several neuroendocrine and inflammatory pathways control energy balance. The parasympathetic nervous system and pathways triggered by insulin, insulin-like growth factor- 1 (REF. 15), androgens, estrogens ${ }^{24}$ and osteocalcin ${ }^{25}$, facilitate the storage of energy-rich substrates. The parasympathetic system amplifies insulin sensitivity and secretion and promotes glycogen storage in the liver ${ }^{26}$. By contrast, the hypothalamic-pituitaryadrenal axis, the sympathetic system, thyroid hormones (such as cortisol ${ }^{27}$ ), glucagon ${ }^{28}$ and growth hormone trigger energy mobilization, that is lipolysis, glycogenolysis and gluconeogenesis ${ }^{19}$.

In addition to promoting energy storage, the parasympathetic system has an important anti-inflammatory effect $^{29}$, which is central to survival as uncontrolled inflammation can be life-threatening. The neuronal acetylcholine receptor subunit $\alpha-7$, which is expressed in the nervous and immune systems, is a key regulator of the inflammatory response ${ }^{29}$ and triggers the cholinergic anti-inflammatory reflex (REF. 30).

The sympathetic system, which is coupled to metabolism by insulin, a well-recognized sympathoexcitatory stimulus $^{31}$, also contributes to the immune response. In stressful conditions, activation of the sympathetic system, which promotes glycogenolysis, gluconeogenesis and lipolysis via its peripheral nervous fibres and the adrenal medulla ${ }^{32}$, helps to fuel the immune system in order to mount an adequate inflammatory response $\mathrm{e}^{15}$. Conversely, sympathetic denervation ablated the early inflammatory response in an experimental model of arthritis ${ }^{33}$.

Stimulation of the two arms of the autonomic system produces opposite effects on energy balance and activation of the immune system. The parasympathetic system is geared towards facilitating energy storage and restraining inflammation, whereas the sympathetic system facilitates energy mobilization and consumption and potentiates inflammation. Overall, the autonomic system, the immune system and metabolism are tightly linked to ensure perfect integration of these systems in homeostasis and life-threatening situations ${ }^{15}$.

\section{Inflammation}

The level of inflammatory mediators increases progressively as renal function declines ${ }^{34}$. Among 3,939 patients enrolled in the Chronic Renal Insufficiency Cohort study, estimated glomerular filtration rate (eGFR), albuminuria and the levels of cystatin $\mathrm{C}$ strongly correlated with the levels of IL-6, tumour necrosis factor (TNF), inverse acute phase reactants such as albumin, and fibrinogen, which mediates the effect of inflammation on the coagulation system ${ }^{35}$. The levels of serum fetuin-A, which is another major inverse acute phase reactant, the most potent circulating inhibitor of calcium phosphorus precipitation and an inhibitor of insulin sensitivity, decline as renal function deteriorates ${ }^{36}$. Inflammation is multifactorial in CKD and this disease is considered to be a prototypical example of inflammatory disease and premature ageing ${ }^{37}$ (FIG. 2). Proinflammatory factors in CKD include reduced cytokine clearance ${ }^{38}$; infections ${ }^{39}$ such as periodontal disease $\mathrm{e}^{40}$; oxidative stress ${ }^{41}$; senescence-associated muscle cell phenotype $^{42}$; hypogonadism ${ }^{43}$; accumulation of advanced glycation end-products ${ }^{44}$ and toxins absorbed in the gut $^{45}$; sodium overload ${ }^{46}$; metabolic acidosis ${ }^{47}$; bone mineral disorders ${ }^{48}$; accumulation of calciprotein particles $^{49}$; autonomic imbalance ${ }^{50}$; insulin resistance ${ }^{51}$; intradialytic hypoxaemia ${ }^{52}$; and genetic ${ }^{53}$ and epigenetic $^{54}$ factors. Thyroid hormone, which regulates thermogenesis by potentiating adrenergic stimulation at the receptor level ${ }^{55}$, is downregulated in advanced 


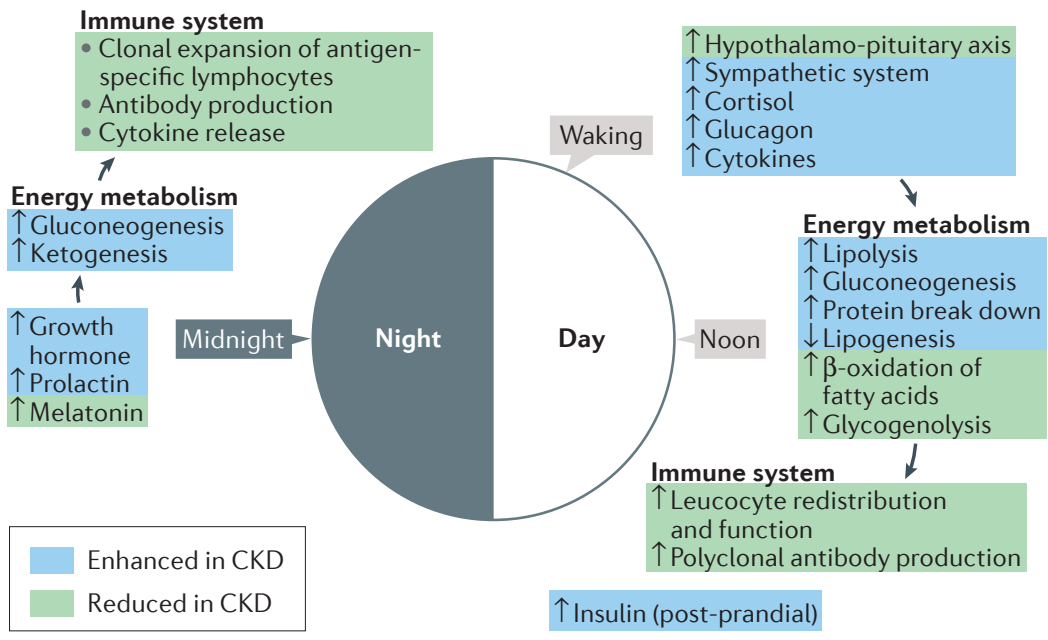

Figure 1 | Circadian changes in energy metabolism and immune responses in CKD. Metabolic and endocrine responses can be promoted or inhibited in patients with chronic kidney disease (CKD). During the circadian cycle in these patients sympathetic activity is markedly increased, whereas the hypothalamic-pituitary axis is dysfunctional. Cortisol and glucagon levels are elevated mainly as a consequence of reduced renal clearance of these hormones and circadian changes in cortisol levels are abolished in this disease. Insulin levels are also high and insulin resistance is a hallmark of CKD. Similarly levels of growth hormone are substantially raised and this change is accompanied by marked resistance to its metabolic effects. Melatonin levels are depressed, particularly during the night, prolactin levels are elevated and the $24 \mathrm{~h}$ rhythmicity of these hormones is disrupted in advanced CKD. Gluconeogenesis is enhanced, whereas glycogenolysis shows an opposite response and these alterations in glucose metabolism are accompanied by reduced $\beta$-fatty acid oxidation and increased lipogenesis and lipolysis. Figure adapted with permission from John Wiley and Sons (C) Straub, R. H. et al., J. Intern. Med. 267, 543-560, (2010).

CKD. This alteration is considered to be a protective mechanism that limits the energy expenditure of the inflammatory response $\mathrm{e}^{5,57}$.

The inflammation-autonomic system interface. High sympathetic activity can trigger and/or modulate inflammation in CKD through multiple pathways such as interferon- $\gamma$, IL- 6 and IL-10 (REF. 58). Dysfunction of the intestinal barrier in CKD can promote the absorption of inflammatory toxins from the gut ${ }^{59,60}$ and can lead to the release of the sympathetic neurotransmitter, neuropeptide $\mathrm{Y}$, a fundamental orchestrator of the link between the gut and the autonomic and central nervous systems $\mathrm{s}^{61}$. The contribution of the gut to the systemic effects of CKD is discussed further below. High levels of proinflammatory cytokines including TNF ${ }^{62}$, IL-6 (REF. 63), leptin ${ }^{64}$, and fairly low levels of adiponectin ${ }^{65}$, regulate insulin sensitivity and energy balance in patients with CKD.

The autonomic system acts as a powerful orchestrator of inflammation and a modulator of energy balance ${ }^{66}$. CKD and high CRP levels are independently associated with an increased risk of cerebrovascular events, whereas CKD and high levels of the sympathetic neurotransmitter noradrenaline have a synergistic effect on the risk of stroke $^{67}$. Chronic inflammation in CKD maintains a catabolic state that leads to sarcopenia and protein-energy wasting $12,68,69$. Common complications of CKD, such as anorexia, cachexia, insulin resistance, dyslipidemia, hypertension, anaemia and high sympathetic activity, can be considered to be consequences of disturbed energy regulation as a result of chronic inflammation ${ }^{19}$. A parasympathetic anti-inflammatory reflex is generated by cytokines in inflamed organs ${ }^{29,30}$, whereas sympathetic activation has a proinflammatory effect ${ }^{33}$. Persistent sympathetic activation can trigger cardiac hypertrophy and fibrosis through activation of the immune system ${ }^{58}$. Accordingly, in patients with stage 3-5CKD, concentric left ventricular hypertrophy is associated with inflammation $^{70,71}$ and with high levels of noradrenaline ${ }^{72}$ and neuropeptide $\mathrm{Y}^{73}$. High levels of these biomarkers underlie high cardiovascular risk in $\mathrm{CKD}^{74,75}$.

Hypoxia. The inflammatory milieu in CKD and other chronic diseases is characterized by tissue hypoxia ${ }^{76}$. Inflammatory stimuli impair nitric oxide synthesis and lead to endothelial-to-mesenchymal transition, which is a key step in capillary rarefaction and ensuing tissue hypoxia in the kidney ${ }^{77}$. The rarefaction of peritubular capillaries is a hallmark of tubulointerstitial alterations, including macrophage infiltration and fibrosis, that accompany the decline in GFR in $\mathrm{CKD}^{78}$. Tubulointerstitial hypoxia and the ensuing activation of hypoxia-inducible transcription factors occurs in the advanced stages of the disease ${ }^{79}$. An imbalance between hypoxia-inducible pro-angiogenic compounds (such as vascular endothelial growth factor (VEGF) and angiopoietins) and angiogenesis inhibitors (such as angiostatin and thrombospondin-1) underlies capillary rarefaction in $\mathrm{CKD}^{79}$. The importance of maintaining a tight balance between pro-angiogenic and anti-angiogenic factors is highlighted by the observation that use of angiogenic factors, such as VEGF, to increase oxygenation of the tubule interstitium results in inflammation and in an increase in vascular permeability and proteinuria, whereas VEGF blockade causes hypertension ${ }^{80}$ and thrombotic microangiopathy ${ }^{81}$.

Oxidative stress and accumulation of advanced glycation end-products can lead to inflammation in CKD and might contribute to adverse renal and cardiovascular outcomes ${ }^{44}$. High levels of reactive oxygen species owing to inflammation have a primary role in the development and/or aggravation of cardiovascular and noncardiovascular comorbidities in $\mathrm{CKD}^{82}$ and in kidney failure ${ }^{41}$. Hypoxia-inducible factor (HIF) (BOX 2) protects the kidney from renal ischaemia and acute kidney injury ${ }^{83}$, but HIF function might be inhibited by oxidative stress and uraemic toxins ${ }^{84}$. Indoxyl sulfate accumulates in CKD and dose-dependently suppresses the transcriptional activity of HIF ${ }^{85}$ and the expression of Klotho ${ }^{86}$, a crucial anti-inflammatory and anti-ageing factor ${ }^{37}$. Ongoing studies are testing whether activation of HIF might have a beneficial effect on maintenance of renal function in models of CKD, such as the remnant kidney model ${ }^{87}$. HIF stabilizers such as prolyl hydroxylase inhibitors improve anaemia in $\mathrm{CKD}^{88}$. Inflammation and oxidative stress could be seen as a trade-off of the hormonal response aimed at countering phosphate accumulation in $\mathrm{CKD}^{89}$. The link between bone mineral disorders and the systemic effects of CKD is discussed below. 
Insulin resistance. Insulin resistance occurs in the early stages of $\mathrm{CKD}^{90,91}$ and is present in almost every patient with end-stage renal disease (ESRD) ${ }^{92}$. The mobilization of energy-rich fuels through activation of neuroendocrine pathways results in high levels of free fatty acids, which lead to insulin resistance in the liver, muscle cells and adipocytes in chronic inflammatory conditions ${ }^{93}$. Insulin resistance is also common in other chronic conditions, such as cancer ${ }^{94}$ and rheumatoid arthritis ${ }^{95}$, and is promoted by altered levels of cytokines, particularly TNF ${ }^{96}$, IL-6 (REF. 97), leptin, adiponectin $^{98}$ and resistin ${ }^{99}$, which are typically seen in chronic inflammation.

In contrast to other cell types, immune cells do not develop insulin resistance. Fairly brief energydemanding inflammatory responses are protective, but in persistent inflammatory states a sustained energy flow to the immune system disrupts the balanced distribution of fuels to various stores and organs ${ }^{19}$ and can result in organ damage, protein depletion, negative caloric balance and metabolic adaptations (such as insulin resistance), disease and disability ${ }^{100,101}$.

Sodium, hypertension and inflammation. The risks of CKD progression ${ }^{102}$ and cardiovascular events ${ }^{103}$ in patients with stage 3-5 CKD are linearly associated with high levels of sodium, the most abundant electrolyte in extracellular fluids. Sodium is involved in inflammation in hypertension and because sodium levels are in part regulated by the kidney, sodium links the kidney ${ }^{104}$ to other systems including the integumentary system ${ }^{105}$.

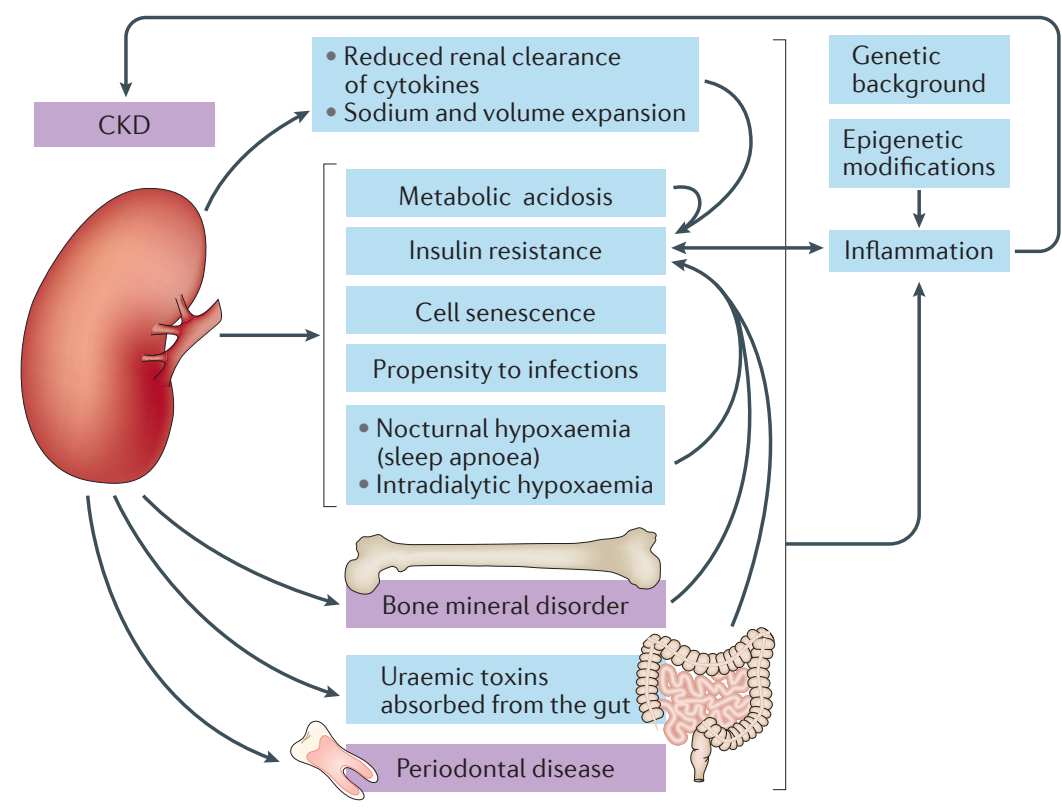

Figure 2 | Proinflammatory mechanisms in CKD. High levels of cytokines, metabolic acidosis, volume and sodium expansion, the presence of uraemic toxins and sleep apnoea (owing to sympathetic overactivity) contribute to insulin resistance in chronic kidney disease (CKD). Proinflammatory mechanisms activated by renal dysfunction might accelerate progression of CKD. Insulin resistance triggers inflammation and vice versa. Genetic and epigenetic factors act as modifiers of endogenous and environmental factors responsible for inflammation in CKD.
For example, a high-salt diet causes interstitial hypertonic accumulation of sodium in the skin and VEGF-C secretion by macrophages. Inhibition of VEGF-C or mononuclear phagocyte system cell depletion increased interstitial hypertonic volume retention, reduced expression of endothelial nitric oxide synthase and caused hypertension in response to high-salt intake ${ }^{106}$.

\section{Metabolism, the bone, the heart and CKD}

Bone mass increases dramatically during growth and preserving skeletal mass during adult life is an absolute functional priority for vertebrates. Phosphate is an anion that is mainly stored in the bone. Given the key role of phosphate in energy metabolism and the structural importance of inorganic phosphate salts in bone, a complex biological system has evolved to link bone to other organs and to adjust phosphate balance to changing physiological requirements and to the availability of nutrients and energy ${ }^{107}$. Phosphate is a fundamental compound for the myocardium and both low $^{108}$ and high phosphate levels ${ }^{109}$ have detrimental effects on the heart. Fibroblast growth factor 23 (FGF-23), the main bonederived hormone that regulates phosphate excretion by the kidney, is involved in myocardial cell growth and in left ventricular hypertrophy ${ }^{110}$.

\section{CKD uncouples mineral and glucose metabolism} Osteocalcin links bone, the sympathetic system and energy metabolism. Osteoblasts secrete osteocalcin, one of the most abundant non-collagen bone proteins. Osteocalcin regulates bone mineralization and modulates osteoblast and osteoclast function. $\gamma$-Carboxylation is essential for osteocalcin biological function and depends on vitamin K levels. Osteocalcin is also a key mediator of the coupling between bone and energy metabolism as it regulates insulin synthesis at the transcriptional level and potently stimulates insulin secretion $^{111}$. Osteocalcin-knockout mice have decreased glucose tolerance, insulin secretion and insulin sensitivity ${ }^{112}$. In humans, the concentration of circulating uncarboxylated forms of osteocalcin depends on vitamin $\mathrm{K}$ status and bone turnover, and vitamin $\mathrm{K}$ levels inversely correlate with $\mathrm{GFR}^{113}$. Of note, in osteoblasts, sympathetic activation enhances the expression of Esp (also known as Ptprv), which encodes osteotesticular protein tyrosine phosphatase, an osteocalcin inhibitor and an insulin secretagogue ${ }^{111}$. These findings highlight the tight relationship between glucose homeostasis and the bone ${ }^{114}$.

In epidemiological and clinical studies, circulating levels of osteocalcin inversely correlated with those of insulin resistance markers (defined according to the Homeostatic Model Assessment of Insulin Resistance criteria) in most, but not all, surveys ${ }^{111}$. Changes in circulating levels of osteocalcin triggered by treatment with risedronate in 87 patients with osteoporosis were not associated with changes in glucose or insulin levels ${ }^{115}$. In three large trials that tested the effects of antiresorptive therapy with alendronate, zeledronic acid or denusomab in postmenopausal women, the treatment, which also reduces osteocalcin levels, did not alter insulin and 
Hypoxia-inducible factor (HIF) regulates over 100 genes involved in erythropoiesis, aerobic and anaerobic metabolism, metabolic reprogramming, angiogenesis, cell proliferation, vascular tone, inflammation, and matrix and barrier function ${ }^{84}$, and is critical for hypoxia-induced responses. HIF prevents pressure overload in chronic heart failure and has a role in metabolic reprogramming in advanced heart failure. This metabolic shift results in a state in which glucose is used as a substrate for glycolytic metabolism and fatty acids are converted to lipids instead of being oxidized to generate ATP, leading to inadequate provision of ATP to sustain cardiac function ${ }^{280}$. Owing to oxidative stress, hyperglycaemia in patients with type 1 diabetes mellitus alters the transactivation of HIF and reduces the expression of vascular endothelial growth factor (VEGF), eventually compromising the potential for neovascularization and tissue repair in this chronic condition ${ }^{281}$. Also owing to oxidative stress, the HIF pathway is suboptimally activated in experimental renal artery stenosis, where defective HIF function and VEGF downregulation results in microvascular remodeling and fibrosis ${ }^{282}$.

glucose levels ${ }^{116}$. Thus, whether osteocalcin has a meaningful effect on glucose metabolism in humans remains unresolved. Differences in osteocalcin genetics, levels, and metabolism between humans and mice could all account for this discrepancy ${ }^{117}$.

In patients with moderate to severe $\mathrm{CKD}$, ostecalcin carboxylation is disrupted owing to vitamin $\mathrm{K}$ deficiency $^{118}$ (FIG. 3). This metabolic alteration can be corrected by vitamin $\mathrm{K} 1$ administration ${ }^{119}$. High levels of uncarboxylated osteocalcin are associated with low levels of plasma glucose, haemoglobin $\mathrm{A}_{1 \mathrm{C}}$, and glycated albuminin in patients with $\mathrm{CKD}^{120}$, suggesting that the lack of carboxylated forms of osteocalcin might contribute to the alteration of glucose metabolism in these patients.

FGF-23 bridges multiple organs and systems. In addition to modulating osteocalcin levels ${ }^{121}$, the sympathetic nervous system regulates the expression of FGF-23 in the bone ${ }^{122}$. FGF-23 regulates energy metabolism (FIG. 3), induces left ventricular hypertrophy ${ }^{123,124}$, and mediates the relationship between the bone and the vascular system. Moreover, FGF-23 is functionally linked to the renin-angiotensin system (RAS); high levels of FGF23 inhibit the expression of angiotensin converting enzyme $2^{125}$ and induce low levels of calcitriol, which can promote renin production.

High circulating levels of FGF-23 are associated with insulin resistance in adolescents with obesity ${ }^{126}$ and correlate with various measures of adiposity including BMI, waist circumference and fat mass in elderly individuals $^{127}$. High levels of this hormone and/or low levels of its co-receptor Klotho are likely causative factors of cardiovascular disease ${ }^{128,129}$ and of progressive loss of renal function in $\mathrm{CKD}^{130-132}$.

FGF-23 (REF. 89) and Klotho ${ }^{133}$ are highly responsive to inflammation ${ }^{19}$. In mouse models of chronic inflammation, the c-terminal portion of FGF-23 is overexpressed, leading to elevated circulating levels of the peptide ${ }^{134}$. Accordingly, the levels of inflammation markers and of C-terminal FGF-23 in the circulation correlate directly in patients with $\mathrm{CKD}^{89}$. In wild-type mice, acute inflammation induced by a single injection of heat-killed Brucella abortus or IL- $1 \beta$ led to increased FGF- 23 cleavage, but the levels of full-length FGF-23, the biologically active form of the hormone, were almost unaltered, presumably owing to FGF-23 overexpression ${ }^{134}$. By contrast, in patients with CKD, the levels of full-length FGF-23 were $50 \%$ lower during acute inflammation secondary to sepsis than after the resolution of this condition, whereas the levels of C-terminal FGF-23 remained unaltered during and after sepsis, potentially owing to suppression of endogenous inhibitors of proteolytic enzymes such as furin pro-pro convertase, which cleave full-length FGF23 during acute sepsis ${ }^{135}$. High levels of FGF-23 irreversibly compromise neutrophil function and recruitment to inflamed tissues ${ }^{136}$.

FGF-23 is also a powerful inhibitor of nitric oxide synthesis ${ }^{137}$. The downregulation of FGF-23 expression in acute inflammatory processes might be an ancestral, life-saving response to acute sepsis. This hypothesis is supported by the simultaneous declines in the levels of FGF-23 and asymmetric dimethyl arginine (ADMA), another potent inhibitor of nitric oxide synthase, that occur during sepsis ${ }^{138}$. These declines result in increased synthesis of nitric oxide, which has a strong bactericidal action. The observation that inhibition of nitric oxide synthase increases mortality in patients with sepsis further supports the hypothesis that unabated nitric oxide production is fundamental for survival in acute sepsis ${ }^{139}$.

Inflammation is profoundly integrated with systems that control energy balance and is a strong modifier of bone resistance to parathyroid hormone ${ }^{140}$, which is a hallmark of ESRD. The inflammatory adipokine leptin and the anti-inflammatory adipokine adiponectin have a major influence on bone mass in experimental models ${ }^{141}$ and leptin is associated with low bone-turnover in kidney failure ${ }^{142}$.

Bone morphogenetic proteins (BMPs). BMPs are members of the transforming growth factor- $\beta$ (TGF- $\beta$ ) superfamily that regulate glucose homeostasis and energy metabolism in the adipose tissue and in the setting of inflammation ${ }^{143}$. BMPs are required for the formation of brown adipocytes and for the browning of white adipose tissue and the related beneficial effects on energy expenditure and adiposity ${ }^{143}$. Polymorphisms in BMP2 seem to have a critical role in inflammation in patients with $\mathrm{CKD}^{144}$. BMP2 stimulates MSX2, which encodes a protein with a role in regulation of bone mass, and enhances phosphate uptake in vascular smooth muscle cells so promotes calcification ${ }^{145}$. BMP2 levels are elevated and might contribute to arterial stiffness in patients with $\mathrm{CKD}^{146}$ (FIG. 3). Some BMPs (such as BMP1 or BMP3) induce kidney fibrosis by binding to TGF- $\beta$ superfamily type II receptors, whereas others (such as BMP7) prevent fibrosis ${ }^{147,148}$. The effects of BMP on these receptors 


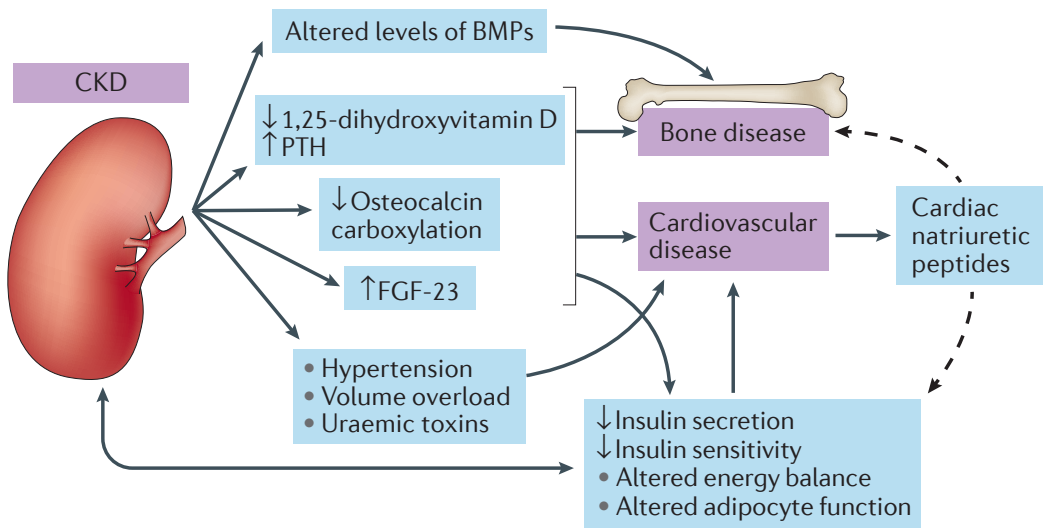

Figure 3 | Energy metabolism regulation, cardiovascular and bone disease in CKD. Altered levels of several molecules such as bone morphogenetic protein (BMP), 1,25-dihydroxyvitamin D, parathyroid hormone (PTH), fibroblast growth factor 23 (FGF-23), insulin, uraemic toxins and cardiac natriuretic peptides, as well as dysfunctional processes such as altered energy balance, adipocyte function, blood pressure and volume control are key risk factors for chronic kidney disease (CKD), bone disease and cardiovascular disease.
Conversely, the uraemic status affects the intestinal microbiome and the integrity of the intestinal wall, which in turn influences toxin generation and inflammatory status, increasing toxicity and hence, systemic complications and mortality in $\mathrm{CKD}^{162,163}$. The potential importance of the gut-kidney link is highlighted by the association between constipation and excess risk of CKD observed in a large cohort of US veterans ${ }^{164}$. Dimethylarginines (ADMA and symmetric dimethylarginine) $)^{165}$ and advanced glycation end-products ${ }^{166}$ originate at least in part from food components in the intestine and have been linked to inflammation ${ }^{44,167}$, CKD progression ${ }^{44,168}$ and all-cause and cardiovascular mortality ${ }^{169-171}$.

Perhaps the most important role of the intestine in the generation of toxic compounds that affect the gutkidney axis (FIG. 4) can be attributed to the breakdown of digestive products such as amino acids by the microbiota into precursors of uraemic retention products, especially indoles and phenyl derivatives ${ }^{172}$. Bacteria that form indole and cresol are predominant in the intestinal microbiome of patients with ESRD ${ }^{162}$, which explains the progressive increase in $p$-cresol levels that occurs in the late stages of $\mathrm{CKD}^{173,174}$. Thus, the intestine produces uraemic toxins and this generation increases as kidney disease progresses. High levels of $p$-cresol and indoxyl sulfate have well-recognized effects on several organs, such as osteoblast dysfunction and low turnover in the bone $\mathrm{e}^{175,176}$ and proinflammatory effects in the vascular system as a result of increased leucocyte adhesion and rolling ${ }^{177}$. Furthermore, both molecules have been linked to cardiovascular diseases and death in $\mathrm{ESRD}^{178}$.

CKD can damage the intestinal wall barrier ${ }^{179}$, increasing endotoxin leakage and translocation of intestinal bacteria, which enhance inflammation and cause cardiovascular and renal damage ${ }^{180}$. Heart failure also increases intestinal leakiness independently of $\mathrm{CKD}^{181}$. Among patients with CKD, intestinal $p$-cresol uptake is higher in those with cardiovascular disease, independent of kidney function ${ }^{182}$. Moreover, the composition of the intestinal microbiome influences the severity of experimental myocardial infarction ${ }^{183}$. Cardiovascular disease might, therefore, impair the function of the intestinal microbiome irrespective of $\mathrm{CKD}$, suggesting that microbial products might have toxic effects in clinical conditions other than CKD.

The intestinal microbiome also generates trimethylamine $N$-oxide (TMAO), a metabolite of dietary phosphatidylcholine and choline ${ }^{184}$. Plasma levels of TMAO increase exponentially with decreasing kidney function $^{185}$ and are associated with mortality and cardiovascular events in $\mathrm{CKD}^{186}$. TMAO could therefore be an intestinally generated cardiotoxic agent that is present at high concentrations in patients with kidney disease. The effects of TMAO might even go beyond the cardiovascular system and extend to bone growth in children ${ }^{187}$, the brain ${ }^{188}$, the endocrine system ${ }^{189}$ and thrombosis ${ }^{190}$.

A 2014 systematic review identified numerous studies that reported deleterious effects of indoxyl sulfate and $p$-cresyl sulfate, such as cardiovascular disease, inflammation and progression of kidney failure ${ }^{178}$. 


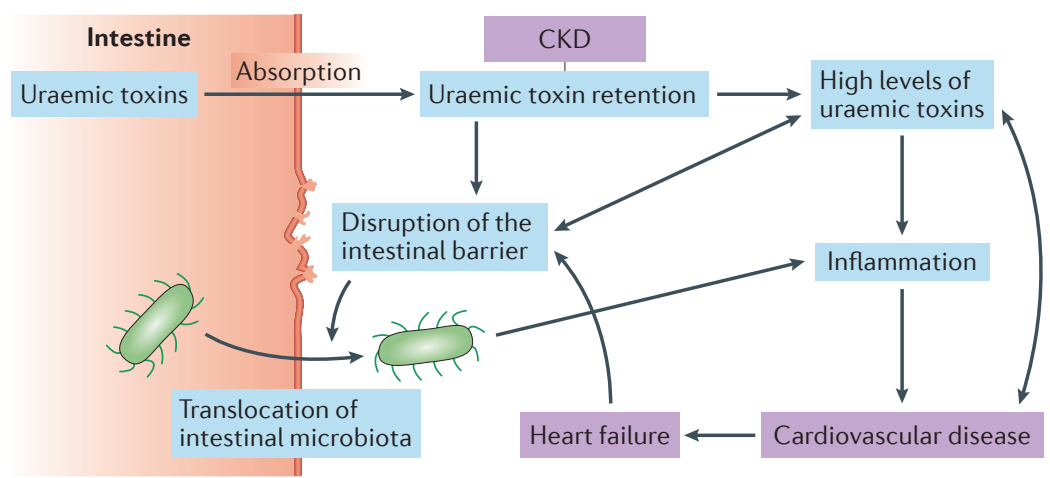

Figure 4 | The gut-kidney axis, inflammation and cardiovascular disease in CKD. Aswell as causing the retention of uraemic toxins (including toxins generated in the intestinal system), chronic kidney disease (CKD) alters the integrity of the intestinal barrier, which leads to translocation of intestinal microbiota into the blood, systemic inflammation, increased absorption of uraemic toxins from the intestine and cardiovascular disease. Uraemic toxins are strong proinflammatory stimuli. Cardiovascular disease also contributes to alter the intestinal barrier via venous intestinal congestion secondary to heart failure.

These toxins also mediate the crosstalk between leukocytes and the vascular wall, a process that is considered to be essential for the initiation of atherogenesis ${ }^{177}$. In a model of acute kidney injury, indoxyl sulfate rapidly accumulated in the nervous system, suggesting that this toxin might be involved in the neural dysregulation that is triggered by kidney dysfunction ${ }^{191}$. Furthermore, accumulating evidence indicates that the gut microbiome might exert important effects on the nervous system through multiple pathways including bacterial metabolites, inflammatory cytokines, and neurotransmitters ${ }^{192}$. Indole acetic acid, which has proinflammatory and pro-coagulatory properties ${ }^{193,194}$, and phenyl acetic acid, an inhibitor of inducible nitric oxide synthase ${ }^{195}$, are also generated in the intestine and have toxic potential.

Symbiotic therapy ${ }^{196}$ (oral intake of a combination of high-molecular weight inulin, fructo-oligosaccharides, galacto-oligosaccharides and a probiotic component including nine different strains across the Lactobacillus, Bifidobacteria and Streptococcus genera) or a reduction in protein intake combined with ketoanalogue administration ${ }^{197}$ (a dietary intervention that can have beneficial effects on renal disease progression and systemic complications of uraemia ${ }^{179}$ ) can be used to mitigate the intestinal generation and uptake of toxic compounds. However, the use of intestinal sorbents to adsorb uraemic toxins did not improve hard outcomes such as progression of kidney failure in patients with CKD stage 3 or $4^{180,181}$. These findings need to be confirmed because toxin concentration was not assessed in one study ${ }^{180}$ and the other could not demonstrate the expected decrease in indoxyl sulfate levels with adsorbent administration ${ }^{181}$.

Overall, the generation and retention of toxic compounds of intestinal origin and the structural changes associated with uraemic and cardiovascular diseases can jeopardize renal, intestinal and cardiovascular integrity by similar pathophysiologic mechanisms
(FIG. 4), resulting in a vicious circle that can lead to complications, hospitalization, and death. As kidney disease progresses, multiorgan damage worsens exponentially and could at least partly explain the exponential increase in mortality that is associated with progression of kidney failure.

\section{Kidney and lung dysfunction}

The lung is constantly exposed to a myriad of changing environmental stimuli and to toxins such as air pollutants, which are risk factors for both lung and kidney disease. For example, ultrafine particles can not only cause lung disease, but also cardiovascular ${ }^{198}$ and renal diseases ${ }^{199}$.

Neuroendocrine cells - an evolutionarily conserved, sparse population of innervated epithelial cells - are essential mediators of the pulmonary response to environmental stimuli ${ }^{181}$. They induce a neural reflex in the lung that regulates cytokine release and modulates inflammation and immune cells function ${ }^{200}$. The contribution of innervation density and neural reflexes to pulmonary inflammatory and immune responses is highlighted by the aggressive alloimmune reaction that follows lung transplantation ${ }^{201}$. In addition, neuroendocrine lung cells are key players in common lung diseases such as chronic obstructive pulmonary disease ${ }^{202}$. Of note, the number of neuroendocrine (calcitonin ${ }^{+}$) cells was considerably increased in the lungs of subtotally nephrectomized rats, compared with control animals ${ }^{203}$. Neuroendocrine cell overpopulation in the lung in patients with advanced CKD might be a compensatory phenomenon to mitigate local inflammation.

The effects of CKD on the risk of obstructive and restrictive lung diseases have received scarce attention to date. In a representative sample of US adults, GFR $<60 \mathrm{~mm} / \mathrm{min} / 1.73 \mathrm{~m}^{2}$ was associated with an increased risk of obstructive lung disease, whereas albuminuria was associated with obstructive and restrictive lung diseases; these relationships were independent of other risk factors ${ }^{204}$. The link between albuminuria and pulmonary disease is most likely multifactorial. Hypoxia might be a major driver of this association as it is a potent activator of the sympathetic system and high sympathetic activity is associated with proteinuria and reduced GFR in patients with $\mathrm{CKD}^{205}$. Proteinuria is more common in people who live at altitudes $>2,400 \mathrm{~m}$ than in those who live at sea level ${ }^{206}$, potentially owing to constant hypoxia. Similarly, in patients with pulmonary disease, hypoxia might cause inflammation and oxidative stress ${ }^{207}$ and thus trigger albuminuria.

In the early stages of acute kidney injury, the lung undergoes inflammation independent of infection, and lung dysfunction is detectable within hours after kidney injury ${ }^{208}$. In patients with ESRD, chronic inflammation and elevated levels of several cytokines exist ${ }^{209}$, and levels of IL- 6 and CRP are very powerful predictors of the risk of death ${ }^{210}$. Patients with ESRD maintained on dialysis have a high risk of emergency hospitalization for pulmonary oedema, and lung congestion in this population is incompletely accounted for by heart failure ${ }^{211}$. This increased risk might be in part due to 
chronic inflammation. Indeed, interstitial accumulation of water in the lung in patients with ESRD is associated with systemic inflammation as reflected by high serum levels of CRP ${ }^{212}$. Notably, pulmonary hypertension, another potential consequence of chronic lung inflammation ${ }^{213}$, is much more frequent in patients with stage $3-5 \mathrm{CKD}^{214,215}$ and in patients undergoing dialysis ${ }^{216}$ than in the general population. As mentioned below, hypoxic episodes during sleep apnoea - a disease characterized by chronic inflammation ${ }^{217}$, persisting sympathetic overactivity ${ }^{218}$ and pulmonary hypertension ${ }^{219}$ - are increasingly common as renal function deteriorates $^{220}$ and evolve to become almost universal in patients maintained on chronic dialysis ${ }^{221}$. Thus, ample evidence demonstrates that the lung and the kidney are linked through neuroendocrine-mediated inflammation and that this relationship is bidirectional.

\section{Neuropathology in CKD}

The kidney and the nervous system are linked by multiple inter-related pathways. For example, a close relationship exists between the sympathetic system, critical renal systems such as the RAS, and the brain ${ }^{222}$. The physiological and behavioural adaptations that have evolved to minimize changes in osmolality also exemplify the interactions between the kidney and the nervous system ${ }^{223}$. Renal excretion of sodium and water counterbalances alterations in the circulating volume, and blood tonicity is maintained in a narrow range by vasopressin (also known as antidiuretic hormone), which is secreted by the nervous system and acts on the kidney ${ }^{224}$. Sensors of blood osmolality (osmoreceptors) are not restricted

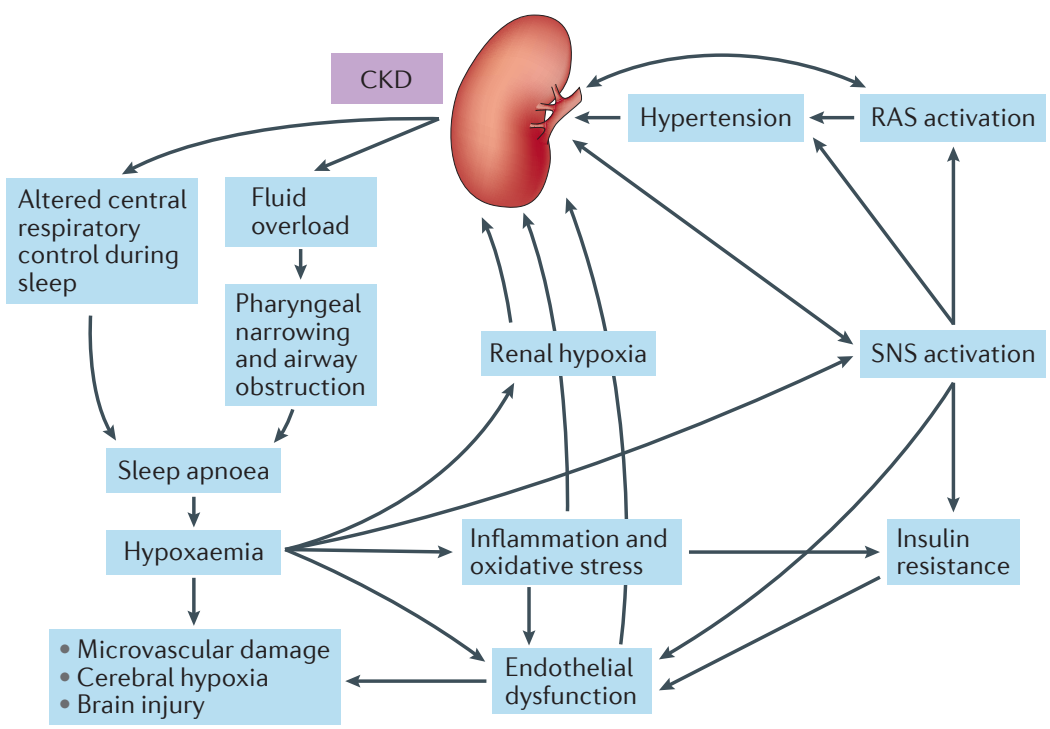

Figure 5 | Inter-relationships between sleep apnoea, CKD and brain injury. In chronic kidney disease (CKD), sleep apnoea is triggered by mechanisms initiated in the central nervous system and by fluid retention leading to pharyngeal narrowing and airway obstruction. Conversely, sleep apnoea can cause CKD via hypoxia and inflammation. Sleep apnoea has major detrimental effects on the cardiovascular system via the development of hypertension, high sympathetic activity, activation of the RAS and aldosterone, insulin resistance, endothelial dysfunction and inflammation.

RAS, renin-angiotensin system; SNS, sympathetic nervous system. to the brain; they are also present in the liver portal vein (peripheral osmoreceptors) where they detect the osmotic effect of foods and fluids entering the gastrointestinal system ${ }^{225}$. Systemic changes in osmolality are detected in critical areas of the central nervous system, including the supraoptic and preoptic nuclei, the subfornical organ, and the organum vasculosum lamina terminalis ${ }^{226}$. Neuronal activity in these areas of the nervous system modifies vasopressin secretion and hence free water clearance, and stimulates or inhibits thirst and sodium appetite ${ }^{226}$.

Another major function of the nervous system in mammals is control of the circadian clock ${ }^{227}$. The suprachiasmatic nucleus in the hypothalamus is the central system that synchronizes peripheral systems ${ }^{227}$. Central and peripheral synchronizers share a common molecular clock that consists of self-sustained transcriptional and translational feedback loops ${ }^{228}$. The circadian regulation of a variety of processes such as sodium excretion, blood pressure control, and circadian neuro-endocrine rhythmicity are notoriously altered in $\mathrm{CKD}^{229}$. The permeability of the blood-brain barrier to small solutes such as sucrose and inulin is enhanced in the advanced stages of CKD and is associated with increased potassium transport and disturbed sodium transport ${ }^{230}$. The relationship between these dysfunctions and altered mental function in CKD remains elusive ${ }^{231}$.

Patients with CKD can have neurological complications such as cognitive disorders ${ }^{232}$, dementia ${ }^{233}$, cerebrovascular diseases ${ }^{234}$ and peripheral neuropathy. Conventional risk factors for cardiovascular disease (such as old age, hypertension, diabetes, dyslipidaemia and atrial fibrillation) might induce neurological disorders in CKD, mainly through microvascular disease. Risk factors associated with low eGFR, including albuminuria ${ }^{235}$, altered NF- $\kappa \mathrm{B}-\mathrm{TNF}$ and Klotho signalling ${ }^{236}$ and increased levels of $\beta 2$-microglobu$\operatorname{lin}^{237}$, are independently associated with structural and functional cerebral changes. In the systemic scenario of CKD, which is characterized by renal and cerebrovascular inflammation, endothelial dysfunction, excess RAS activation and oxidative stress ${ }^{234}$, the levels of a substantial number of neuromediators and endocrine factors (such as $\beta$-endorphin, methionine-enkephalin, adrenomedullin and neuropeptide Y) are temporally (circadian rhythms) and quantitatively altered ${ }^{229,238}$. These changes are thought to have a role in the high incidence of neurological complications in CKD, but this hypothesis remains to be tested.

Sleep apnoea is one of the most common complications in patients with advanced CKD (FIG. 5). Conversely, sleep apnoea can cause CKD, as clearly shown by large two community-based studies ${ }^{239,240}$. In the first study, which included 4,674 patients with and 23,370 individuals without sleep apnoea, this condition was associated with a $94 \%$ excess risk of CKD (HR 1.94; 95\% CI, 1.52-2.46) and a 120\% excess risk of ESRD (HR 2.2; 95\% CI, 1.31-3.69; $\mathrm{P}<0.01$ ) ${ }^{239}$. In the second study, which included $>3$ million US veterans, patients with untreated sleep apnoea had a $127 \%$ (HR 2.27; 95\% CI 2.19-2.36) excess risk of ESRD, 
whereas the same risk was $179 \%$ higher (HR 2.79, 95\% CI 2.48-3.13) in patients receiving treatment for sleep apnoea than in patients without sleep apnoea ${ }^{240}$.

Sleep apnoea has a wide-range of systemic effects including inflammation, oxidative stress, insulin resistance, pulmonary hypertension, left ventricular dysfunction and hypertension, which are typically associated with $\mathrm{CKD}^{241}$. The circadian pattern of the blood pressure profile is often inverted in patients with kidney failure and nocturnal hypoxaemia ${ }^{242}$. Studies in animal models and clinical studies show that sleep apnoea causes neurodegeneration via hypoxia, hypertension, vascular dysfunction, inflammation, and oxidative stress ${ }^{243}$. Moreover, serum amyloid levels are more than doubled in patients with moderate to severe sleep apnoea compared with those with mild sleep apnoea or healthy controls ${ }^{244}$. Well before the appearance of any neuropsychological symptoms, patients with sleep apnoea have grey matter loss in the frontal and temporo-parieto-occipital cortices, the thalamus, the hippocampal region, some basal ganglia and cerebellar regions ${ }^{245}$. Brain alterations and grey matter loss are commonly seen in CKD, particularly in patients with $\mathrm{ESRD}^{246}$. These alterations are clinically relevant as they are associated with cognitive impairment and with a high risk of death in $\mathrm{CKD}^{247}$.

Overall, the kidney has an important influence on the complex neural network that regulates essential functions such as thirst and water balance, sodium appetite and excretion, and circadian rhythms. CKD alters sodium and water metabolism, cerebrovascular function and several neurohormonal signalling mechanisms, which eventually lead to and/or aggravate various neurological complications of CKD spanning from sleep apnoea to peripheral neuropathy and dementia.

\section{Next steps: a systems biology approach A whole body perspective}

From a whole-body perspective, the multiple links between the kidney and other organs generate a complex set of physiological and pathological interactions that are difficult to understand through the study of bilateral interactions between two organs. For example, the links between the kidney and the lungs in sleep apnoea are insufficient to explain the multifaceted pathophysiology of this syndrome, which is characterized by systemic inflammation, nocturnal hypertension, cardiovascular dysfunction and brain dysfunction. Systems biology approaches can provide further insights into the systemic nature of disease $^{248}$. Unraveling multiple pathophysiologic associations between organs in CKD is now a concrete possibility (TABLE 1). However, integrating the large quantity of information that will be generated by the systems biology approach will require technical advances such as those seen in imaging and network analysis. These tools could be generated with research efforts such as those deployed by the United States Brain Research through Advancing Innovative Neurotechnologies Initiative ${ }^{249}$.

\section{A systems biology approach}

In the current post-genomic era, systems biology has the potential to enable in-depth investigation of the nexus between molecular function at the cellular and subcellular levels and whole-body integration. Understanding of

Table 1 | Examples of the application of a systems biology approach to CKD

\begin{tabular}{|c|c|c|c|c|c|c|}
\hline Approach* & $\begin{array}{l}\text { Sample } \\
\text { (species) }\end{array}$ & Tool & Data analyzed & Key finding & Potential implications & Refs \\
\hline Epigenomics & $\begin{array}{l}\text { Monocytes } \\
\text { (human) }\end{array}$ & NGS & $\begin{array}{l}\text { Epigenetic changes } \\
\text { (histone modifications, } \\
\text { DNA modifications } \\
\text { that respect the } \\
\text { sequence of base pairs, } \\
\text { miRNA presence) }\end{array}$ & $\begin{array}{l}68 \text { miRNAs are } \\
\text { differentially expressed in } \\
\text { CKD and connected to } 47 \\
\text { reciprocally differentially } \\
\text { expressed target genes }\end{array}$ & $\begin{array}{l}\text { Potential novel therapeutic targets } \\
\text { for cardiovascular complications in } \\
\text { CKD }\end{array}$ & 250 \\
\hline Proteomics & $\begin{array}{l}\text { Plasma } \\
\text { (human) }\end{array}$ & MS & $\begin{array}{l}\text { Proteins or } \\
\text { peptides, including } \\
\text { post-translational } \\
\text { modifications (such as } \\
\text { phosphorylation) }\end{array}$ & $\begin{array}{l}\text { Identification of } 13 \text { novel } \\
\text { biomarkers of vascular } \\
\text { disease in patients with } \\
\text { CKD }\end{array}$ & $\begin{array}{l}\text { Functional studies that explored the } \\
\text { causal relationship between vascular } \\
\text { disease and CKD might identify new } \\
\text { therapeutic targets }\end{array}$ & 253 \\
\hline Metagenomics & Stool (mice) & NGS & $\begin{array}{l}\text { Coecal microbial } \\
\text { composition in mice }\end{array}$ & $\begin{array}{l}\text { L-Carnitine } \\
\text { supplementation increases } \\
\text { bacteria taxa that } \\
\text { metabolize L-carnitine to } \\
\text { TMAO precursors }\end{array}$ & $\begin{array}{l}\text { L-Carnitine supplementation in CKD } \\
\text { might promote TMAO accumulation } \\
\text { and platelet hyper-reactivity leading } \\
\text { to increased thrombosis risk }\end{array}$ & $\begin{array}{r}190, \\
258\end{array}$ \\
\hline
\end{tabular}


how the function of a myriad of proteins translates into the complex and integrated activity of cells, tissues and organs systems, and how altered molecular regulation of these activities by CKD might lead to organ dysfunction, is now possible.

A systems biology approach enables simultaneous analysis of the full set of molecular changes occurring at different levels and in different organs. The epigenome can provide insights into the effects of uraemia and the resulting inflammation on heritable modifications of DNA, histones and microRNAs that modulate gene expression without modifying the DNA sequence ${ }^{250}$. For example, kidney-injury-associated inflammation decreases Klotho expression in part through epigenetic modulation ${ }^{251}$. Analyses of the transcriptome enable the detection of detailed changes in gene expression in CKD to refine the molecular definition of $\mathrm{CKD}$-associated inflammation and organ dysfunction ${ }^{252}$.

Our understanding of uraemic toxicity can also be furthered by a systems biology approach. At the proteomic level, differential levels of protein expression ${ }^{253}$ and post-translational protein modifications resulting from altered gene expression or uraemic toxicity can be precisely defined, setting the stage for novel therapeutic approaches. For example, post-translational modifications that change protein half-life or function, such as carbamylation, or high levels of methylglyoxal-derived advanced glycation end-products have been identified in patients with uraemia ${ }^{254}$.

\section{Metabolomics and metagenomics}

Changes in the levels of metabolites, such as asymmetric dimethyl arginine ${ }^{255}$ or long-chain acylcarnitine ${ }^{256}$, that contribute to complications in CKD and are involved in cardiovascular complications in patients undergoing dialysis ${ }^{169,256}$ can be reliably measured and are providing new insights into the pathogenesis of complications associated with uraemia ${ }^{255-257}$. Studies at the metagenomic level, that is on genetic material from bacterial communities such as the gut microbiota, provide insights into the effects of CKD on the microbiome and, conversely, on the effects of changes in the microbiome on the manifestations of CKD. Metagenomic studies in mice have shown that the metabolism of dietary L-carnitine (which is abundant in red meat) by certain taxa of the intestinal microbiota generates trimethylamine, which is processed by the liver into pro-atherogenic and prothrombotic trimethylamine-N-oxide ${ }^{190,258}$. Changes in the gut flora induced by diet and uraemia-associated trimethylamine-N-oxide accumulation ${ }^{259}$ modify the manifestations of CKD and might underlie the clinical variability and discrepancy seen in the results of different studies ${ }^{260}$.

\section{Integration of omic sciences}

The possibility that the complexity of renal diseases could be unraveled by studies based on new systems biology technologies is tantalizing because full understanding of pathophysiological processes requires the integration of several omics datasets (from biological fluids or tissues) into a dynamic model of the molecular changes in
CKD through 'trans-omic' analyses ${ }^{261}$. In this regard, the process has started with the application of systems biology tools to simple sets of samples (which can be obtained from plasma, urine, faeces or circulating leukocytes). For example, one study performed metabolomics analyses in a group of 41 patients with stage $3-4 \mathrm{CKD}^{255}$, whereas another analysed metabolic signatures in urine samples of a small series of 13 patients with stage $4-5 \mathrm{CKD}^{257}$.

In the future, the complexity of the samples can be expected to increase. Indeed, understanding of the effects of CKD and uraemia on the epigenome, transcriptome, proteome and metabolome of individual organs such as the heart will require additional tissue analyses with techniques such as imaging, which enables visualization of the heart structure. These analyses will also have to be integrated with the genetic background of the individual, which can be studied by exome or whole genome sequencing. Progress in our understanding of the pathogenesis and the manifestations of CKD and the uraemic syndrome is likely to be a slow, decades-long process. The full development of new informatic and biostatistical tools to integrate the large amount of biological data generated by the various omics sciences, from genomics ${ }^{262}$ to metabolomics ${ }^{263}$, will be central for the advancement of knowledge on CKD.

\section{Personalized renal care}

To achieve the ambition of personalized care for patients with renal diseases, understanding of not only how renal diseases arise, but also how they affect all organs at the molecular and individual level would be useful. Developing such a comprehensive view of renal diseases and generating practical clinical applications of this knowledge might take at least $10-15$ years $^{264}$. Such an undertaking seems worthwhile if one considers the uraemic syndrome and CKD with a holistic perspective and recognizes that many systems are intertwined; unravelling all the links in the network will, therefore, likely lead to improvements in patient outcomes.

Treatments that target critical integrative pathways such as inflammation and the autonomic system might mitigate the systemic effects and progression of CKD. To date attempts to interfere with these systems have been disappointing ${ }^{265}$, but thorough proteomic and metabolomic analyses might lead to the discovery of novel critical biological targets for therapy and the development of innovative drugs with increased efficacy and reduced adverse effects.

Systems biology tools such as proteomics evolve rapidly and can be applied to the investigation of the biology of chronic diseases. Proteomics has proven to be an effective tool to identify drug targets in cancer, a chronic condition that involves extensive protein networks ${ }^{266}$, much like $\mathrm{CKD}^{267}$. In the specific context of $\mathrm{CKD}$, rich databases that combine findings at various biological levels have already been created $^{268}$. These databases, which will potentially be fuelled by future discoveries, will soon provide critical information for our understanding of the complexity of CKD and its systemic complications, thereby enabling the discovery of novel biomarkers and therapeutic targets ${ }^{268}$. 


\section{Conclusions}

Traditionally, medicine aims to isolate the most relevant single factor responsible for a disease: a bacterium or a virus in an infectious process, a gastroduodenal ulcer in anaemia due to blood loss, the tumour mass in cancer ${ }^{248}$. Such an approach has been and remains crucial but, in the context of multifactorial, complex diseases, failing to capture critical multiorgan information is inherently inadequate. Until recently, the analytical tools available for medical research were not adapted to addressing complex questions. In kidney diseases, we define syndromes such as the cardiorenal syndrome, cardiorenal anaemia, and CKD-MBD ${ }^{12,13}$, but $\mathrm{CKD}^{269}$ and uraemia ${ }^{270}$ are systemic diseases. With emerging omic sciences and personalized medicine, CKD syndromes built on uncertain grounds might soon be considered just as descriptive expressions of this multifaceted disease.
1. KDIGO. KDIGO 2012 clinical practice guideline for the evaluation and management of chronic kidney disease. Kidney Int. Suppl. 3, 19-62 (2013).

2. Eckardt, K.-U. et al. Evolving importance of kidney disease: from subspecialty to global health burden. Lancet 382, 158-169 (2013).

3. MacCluer, J. W. et al. Heritability of measures of kidney disease among Zuni Indians: the Zuni Kidney Project. Am. J. Kidney Dis. 56, 289-302 (2010).

4. Barrett Bowling, C. et al. Age-specific associations of reduced estimated glomerular filtration rate with concurrent chronic kidney disease complications Clin. J. Am. Soc. Nephrol. 6, 2822-2828 (2011).

5. Zoccali, C., Bolignano, D. \& Mallamaci, F. in Oxord Textbook of Clinical Nephrology 4th edn Ch. 107 (eds Turner, N. N. et al.) 837-852 (Oxford Univ. Press, 2015)

6. Maarouf, O. H. et al. Paracrine Wnt 1 drives interstitial fibrosis without inflammation by tubulointerstitial cross-talk. J. Am. Soc. Nephrol. 27 781-790 (2015)

7. Abbas, N. A. et al. Cardiac troponins and renal function in nondialysis patients with chronic kidney disease. Clin. Chem. 51, 2059-2066 (2005)

8. Vickery, S. et al. B-type natriuretic peptide (BNP) and amino-terminal proBNP in patients with CKD: relationship to renal function and left ventricular hypertrophy. Am. J. Kidney Dis. 46, 610-620 (2005).

9. Tang, W. H. W. et al. Usefulness of plasma galectin-3 levels in systolic heart failure to predict renal insufficiency and survival. Am. J. Cardiol. 108, 385-390 (2011)

10. Evenepoel, P., D'Haese, P. \& Brandenburg, V. Sclerostin and DKK 1: new players in renal bone and vascular disease. Kidney Int. 88, 235-240 (2015).

11. Ketteler, M. et al. Revisiting KDIGO clinical practice guideline on chronic kidney disease - mineral and bone disorder: a commentary from a Kidney Disease: Improving Global Outcomes controversies conference Kidney Int. 87, 502-528 (2015).

12. Zoccali, C. et al. The complexity of the cardio-renal link: taxonomy, syndromes, and diseases. Kidney Int. Suppl. 1, 2-5 (2011).

13. Cozzolino, M. et al. Is chronic kidney disease-mineral bone disorder (CKD-MBD) really a syndrome? Nephrol. Dial. Transplant. 29, 1815-1820 (2014).

14. Brigandt, I. \& Love, A. in The Stanford Encyclopedia of Philosophy (ed. Zalta, E. N.) (Stanford Univ., 2017).

15. Macdougall, I. C. et al. Beyond the cardiorenal anaemia syndrome: recognizing the role of iron deficiency. Eur. J. Heart Fail. 14, 882-886 (2012).

16. Beresford, M. J. Medical reductionism: lessons from the great philosophers. OJM 103, 721-724 (2010).

17. Hektoen International. Richard Bright, the father of nephrology. HekInt.org http://www. hektoeninternational.org/index.php?option $=\mathrm{com}$ content\&view = article£id = 705 (2013)

18. Seldin, D. W., Carter, N. W. \& Rector, F. C. in Strauss and Welt's Diseases of the Kidney Vol. 2 (eds Strauss, M. B. \& Welt, L. G.) 211-272 (Little Brown Co., 1971).

19. Straub, R. H., Cutolo, M., Buttgereit, F. \& Pongratz, G. Energy regulation and neuroendocrine-immune control in chronic inflammatory diseases. J. Intern. Med. 267, 543-560 (2010)

20. Manning, P. J. et al. Postprandial cytokine concentrations and meal composition in obese and lean women. Obesity (Silver Spring) 16, 2046-2052 (2008)

21. Walford, R. L., Harris, S. B. \& Gunion, M. W. The calorically restricted low-fat nutrient-dense diet in Biosphere 2 significantly lowers blood glucose, tota leukocyte count, cholesterol, and blood pressure in humans. Proc. Natl Acad. Sci. USA 89, 11533-11537 (1992).
22. Maciver, N. J. et al. Glucose metabolism in lymphocytes is a regulated process with significant effects on immune cell function and survival. J. Leukoc. Biol. 84, 949-957 (2008).

23. Frauwirth, K. A. \& Thompson, C. B. Regulation of T lymphocyte metabolism. J. Immunol. 172 4661-4665 (2004).

24. Mauvais-Jarvis, F. Estrogen and androgen receptors: regulators of fuel homeostasis and emerging targets for diabetes and obesity. Trends Endocrinol. Metab. 22, 24-33 (2011).

25. Ducy, P. The role of osteocalcin in the endocrine cross-talk between bone remodelling and energy metabolism. Diabetologia 54, 1291-1297 (2011).

26. Moore, M. C. et al. Effect of hepatic denervation on peripheral insulin sensitivity in conscious dogs. Am. J. Physiol. Endocrinol. Metab. 282, E286-E296 (2002).

27. Christiansen, J. J. et al. Effects of cortisol on carbohydrate, lipid, and protein metabolism: studies of acute cortisol withdrawal in adrenocortical failure. J. Clin. Endocrinol. Metab. 92, 3553-3559 (2009).

28. Jones, B. J., Tan, T. \& Bloom, S. R. Minireview: glucagon in stress and energy homeostasis. Endocrinology 153, 1049-1054 (2012).

29. Báez-Pagán, C. A., Delgado-Vélez, M. \& Lasalde Dominicci, J. A. Activation of the macrophage $\alpha 7$ nicotinic acetylcholine receptor and control of inflammation. J. Neuroimmune Pharmacol. 10, 468-476 (2015)

30. Andersson, U. \& Tracey, K. J. Neural reflexes in inflammation and immunity. J. Exp. Med. 209, 1057-1068 (2012)

31. Scherrer, U. \& Sartori, C. Insulin as a vascular and sympathoexcitatory hormone: implications for blood pressure regulation, insulin sensitivity, and cardiovascular morbidity. Circulation 96, 4104-4113 (1997).

32. Rui, L. Energy metabolism in the liver. Compr. Physiol. 4, 177-197 (2014)

33. Härle, P., Möbius, D., Carr, D. J. J., Schölmerich, J. \& Straub, R. H. An opposing time-dependent immunemodulating effect of the sympathetic nervous system conferred by altering the cytokine profile in the local lymph nodes and spleen of mice with type II collagen induced arthritis. Arthritis Rheum. 52, 1305-1313 (2005)

34. Mehrotra, R. et al. Serum fetuin-A in nondialyzed patients with diabetic nephropathy: relationship with coronary artery calcification. Kidney Int. 67 1070-1077 (2005)

35. Gupta, J. et al. Association between albuminuria, kidney function, and inflammatory biomarker profile in CKD in CRIC. Clin. J. Am. Soc. Nephrol. 7 1938-1946 (2012).

36. Cottone, S. et al. Relationship of fetuin-A with glomerular filtration rate and endothelial dysfunction in moderate-severe chronic kidney disease. J. Nephrol. 23, 62-69 (2010)

37. Stenvinkel, P. \& Larsson, T. E. Chronic kidney disease: a clinical model of premature aging. Am. J. Kidney Dis. 62, 339-351 (2013).

38. Schindler, R. Causes and therapy of microinflammation in renal failure. Nephrol. Dial. Transplant. 19, 34-40 (2004).

39. Levey, A. S. et al. Chronic kidney disease as a global public health problem: approaches and initiatives - a position statement from Kidney Disease Improving Global Outcomes. Kidney Int. 72, 247-259 (2007)

40. Akar, H., Akar, G. C., Carrero, J. J., Stenvinkel, P. \& Lindholm, B. Systemic consequences of poor oral health in chronic kidney disease patients. Clin. J. Am. Soc. Nephrol. 6, 218-226 (2011).
41. Locatelli, F. et al. Oxidative stress in end-stage rena disease: an emerging threat to patient outcome Nephrol. Dial. Transplant. 18, 1272-1280 (2003).

42. Gardner, S. E., Humphry, M., Bennett, M. R. \& Clarke, M. C. H. Senescent vascular smooth muscle cells drive inflammation through an interleukin$1 \alpha$-dependent senescence-associated secretory phenotype. Arterioscler. Thromb. Vasc. Biol. 35, 1963-1974 (2015).

43. Carrero, J. J. et al. Low serum testosterone increases mortality risk among male dialysis patients. $\mathrm{J}$. Am. Soc. Nephrol. 20, 613-620 (2009).

44. Vlassara, H. et al. Role of oxidants/inflammation in declining renal function in chronic kidney disease and normal aging. Kidney Int. Suppl. 76, S3-S11 (2009).

45. Sun, C.-Y., Hsu, H.-H. \& Wu, M.-S. p-Cresol sulfate and indoxyl sulfate induce similar cellular inflammatory gene expressions in cultured proximal renal tubular cells. Nephrol. Dial. Transplant. 28, 70-78 (2013).

46. Rao, A. K. et al. Left atrial volume is associated with inflammation and atherosclerosis in patients with kidney disease. Echocardiography 25, 264-269 (2008).

47. de Nadai, T. R. et al. Metabolic acidosis treatment as part of a strategy to curb inflammation. Int. J. Inflam. 2013, 601424 (2013).

48. Viaene, L. et al. Inflammation and the bone-vascular axis in end-stage renal disease. Osteoporos. Int. 27, 489-497 (2016).

49. Aghagolzadeh, P. et al. Calcification of vascular smooth muscle cells is induced by secondary calciprotein particles and enhanced by tumor necrosis factor- $\alpha$. Atherosclerosis 251, 404-414 (2016).

50. Underwood, C. F. et al. Uraemia: an unrecognized driver of central neurohumoral dysfunction in chronic kidney disease? Acta Physiol. (Oxf.) 219, 305-323 (2016).

51. Thomas, S. S, Zhang L. \& Mitch W. E. Molecular mechanisms of insulin resistance in chronic kidney disease. Kidney Int. 88, 1233-1239 (2015).

52. Meyring-Wösten, A. et al. Intradialytic hypoxemia and clinical outcomes in patients on hemodialysis. Clin. J. Am. Soc. Nephrol. 11, 616-625 (2016)

53. Spoto, B. et al. Association of IL-6 and a functional polymorphism in the IL- 6 gene with cardiovascular events in patients with CKD. Clin. J. Am. Soc. Nephrol. 10, 232-240 (2015)

54. Stenvinkel, P. et al. Impact of inflammation on epigenetic DNA methylation - a novel risk factor for cardiovascular disease? J. Intern. Med. 261, 488-499 (2007)

55. Silva, J. E. Thyroid hormone control of thermogenesis and energy balance. Thyroid 5, 481-492 (1995).

56. Zoccali, C., Tripepi, G., Cutrupi, S., Pizzini, P. \& Mallamaci, F. Low triiodothyronine: a new facet of inflammation in end-stage renal disease. J. Am. Soc Nephrol. 16, 2789-2795 (2005).

57. Meuwese, C. L., Dekkers, O. M., Stenvinkel, P., Dekker, F. W. \& Carrero, J. J. Nonthyroidal illness and the cardiorenal syndrome. Nat. Rev. Nephrol. 9 599-609 (2013)

58. Levick, S. P., Murray, D. B., Janicki, J. S. \& Brower, G. L. Sympathetic nervous system modulation of inflammation and remodeling in the hypertensive heart. Hypertension 55, 270-276 (2010).

59. Mafra, D. et al. Role of altered intestinal microbiota in systemic inflammation and cardiovascular disease in chronic kidney disease. Future Microbiol. 9, 399-410 (2014).

60. Koppe, L., Mafra, D. \& Fouque, D. Probiotics and chronic kidney disease. Kidney Int. 88, 1-9 (2015)

61. Holzer, P. \& Farzi, A. Neuropeptides and the microbiota-gut-brain axis. Adv. Exp. Med. Biol. 817 195-219 (2014) 
62. Lai, H. L., Kartal, J. \& Mitsnefes, M. Hyperinsulinemia in pediatric patients with chronic kidney disease: the role of tumor necrosis factor-a. Pediatr. Nephrol. 22, 1751-1756 (2007).

63. Landau, M. et al. Correlates of insulin resistance in older individuals with and without kidney disease. Nephrol. Dial. Transplant. 26, 2814-2819 (2011).

64. Mallamaci, F., Tripepi, G. \& Zoccali, C. Leptin in end stage renal disease (ESRD): a link between fat mass, bone and the cardiovascular system. J. Nephrol. 18, 464-468 (2005)

65. Yoo, D. E. et al. Low circulating adiponectin levels are associated with insulin resistance in non-obese peritoneal dialysis patients. Endocr. J. 59, 685-695 (2012).

66. Landsberg, L. \& Young, J. B. Fasting, feeding and regulation of the sympathetic nervous system. N. Engl. J. Med. 298, 1295-1301 (1978).

67. Yano, Y. et al. Synergistic effect of chronic kidney disease and high circulatory norepinephrine level on stroke risk in Japanese hypertensive patients. Atherosclerosis 219, 273-279 (2011).

68. Fouque, D. et al. A proposed nomenclature and diagnostic criteria for protein-energy wasting in acute and chronic kidney disease. Kidney Int. 73, 391-398 (2008).

69. Kalantar-Zadeh, K., Ikizler, T. A., Block, G. Avram, M. M. ¿ Kopple, J. D. Malnutritioninflammation complex syndrome in dialysis patients: causes and consequences. Am. J. Kidney Dis. 42 , 864-881 (2003)

70. Gupta, J. et al. Association between inflammation and cardiac geometry in chronic kidney disease: findings from the CRIC study. PLoS ONE 10, e0124772 (2015)

71. Zoccali, C. et al. Fibrinogen, inflammation and concentric left ventricular hypertrophy in chronic renal failure. Eur. J. Clin. Invest. 33, 561-566 (2003).

72. Zoccali, C. et al. Norepinephrine and concentric hypertrophy in patients with end-stage renal disease. Hypertension 40, 41-46 (2002)

73. Zoccali, C. et al. Neuropeptide Y, left ventricular mass and function in patients with end stage renal disease. J. Hypertens. 21, 1355-1362 (2003).

74. Zoccali, C et al. Plasma norepinephrine predicts survival and incident cardiovascular events in patients with end-stage renal disease. Circulation 105, 1354-1359 (2002).

75. Zoccali, C. et al. Prospective study of neuropeptide $Y$ as an adverse cardiovascular risk factor in end-stage renal disease. J. Am. Soc. Nephrol. 14, 2611-2617 (2003).

76. Eltzschig, H. K. \& Carmeliet, P. Hypoxia and inflammation. N. Engl. J. Med. 364, 656-665 (2011).

77. O'Riordan, E. et al. Chronic NOS inhibition actuates endothelial-mesenchymal transformation. Am. J. Physiol. Heart Circ. Physiol. 292, H285-H294 (2007).

78. Kang, D.-H. et al. Role of the microvascular endothelium in progressive renal disease. J. Am. Soc Nephrol. 13, 806-816 (2002).

79. Tanaka, T. \& Nangaku, M. Angiogenesis and hypoxia in the kidney. Nat. Rev. Nephrol. 9, 211-222 (2013).

80. Hussain, S. et al. Outcome among patients with acute renal failure needing continuous renal replacement therapy: a single center study. Hemodial. Int. 13, 205-214 (2009)

81. Bollée, G. et al. Thrombotic microangiopathy secondary to VEGF pathway inhibition by sunitinib. Nephrol. Dial. Transplant. 24, 682-685 (2009).

82. Massy, Z. A., Stenvinkel, P. \& Drueke, T. B. The role of oxidative stress in chronic kidney disease. Semin. Dial. 22, 405-408 (2009)

83. Kapitsinou, P. P. \& Haase, V. H. Molecular mechanisms of ischemic preconditioning in the kidney. Am. J. Physiol. Renal Physiol. 309, F821-F834 (2015).

84. Tanaka, T. Expanding roles of the hypoxia-response network in chronic kidney disease. Clin. Exp. Nephrol. 20, 835-844 (2016).

85. Tanaka, T., Yamaguchi, J., Higashijima, Y. \& Nangaku, M. Indoxyl sulfate signals for rapid mRNA stabilization of $\mathrm{Cbp} / \mathrm{p} 300$-interacting transactivator with Glu/Asp-rich carboxy-terminal domain 2 (CITED2) and suppresses the expression of hypoxia-inducible genes in experimental CKD and uremia. FASEB J. 27 4059-4075 (2013).

86. Sun, C.-Y., Chang, S.-C. \& Wu, M.-S. Suppression of Klotho expression by protein-bound uremic toxins is associated with increased DNA methyltransferase expression and DNA hypermethylation. Kidney Int 81, 640-650 (2012).
87. Deng, A. et al. Renal protection in chronic kidney disease: hypoxia-inducible factor activation versus angiotensin II blockade. Am. J. Physiol. Renal Physiol. 299, F1365-F1373 (2010).

88. Maxwell, P. H. \& Eckardt, K.-U. HIF prolyl hydroxylase inhibitors for the treatment of renal anaemia and beyond. Nat. Rev. Nephrol. 12, 157-168 (2015).

89. Munoz Mendoza, J. et al. Fibroblast growth factor 23 and Inflammation in CKD. Clin. J. Am. Soc. Nephrol. 7, 1155-1162 (2012)

90. Fliser, D. et al. Insulin resistance and hyperinsulinemia are already present in patients with incipient renal disease. Kidney Int. 53, 1343-1347 (1998).

91. de Boer, I. H. et al. Impaired glucose and insulin homeostasis in moderate-severe CKD. J. Am. Soc. Nephrol. 27, 2861-2871 (2016).

92. DeFronzo, R. A. et al. Insulin resistance in uremia. J. Clin. Invest. 67, 563-568 (1981)

93. Schenk, S., Saberi, M. \& Olefsky, J. M. Insulin sensitivity: modulation by nutrients and inflammation. J. Clin. Invest. 118, 2992-3002 (2008).

94. Djiogue, S. et al. Insulin resistance and cancer: the role of insulin and IGFs. Endocr. Relat. Cancer 20, R1-R17 (2013).

95. Giles, J. T. et al. Insulin resistance in rheumatoid arthritis: disease-related indicators and associations with the presence and progression of subclinical atherosclerosis. Arthritis Rheumatol. 67, 626-636 (2015).

96. Hotamisligil, G. S. et al. IRS-1-mediated inhibition of insulin receptor tyrosine kinase activity in TNF-alphaand obesity-induced insulin resistance. Science 271, 665-668 (1996).

97. Senn, J. J., Klover, P. J., Nowak, I. A. \& Mooney, R. A Interleukin-6 induces cellular insulin resistance in hepatocytes. Diabetes 51, 3391-3399 (2002).

98. Yadav, A., Kataria, M. A., Saini, V. \& Yadav, A. Role of leptin and adiponectin in insulin resistance. Clin. Chim. Acta 417, 80-84 (2013)

99. Muse, E. D. et al. Role of resistin in diet-induced hepatic insulin resistance. J. Clin. Invest. 114 232-239 (2004)

100. Straub, R. H. \& Besedovsky, H. O. Integrated evolutionary, immunological, and neuroendocrine framework for the pathogenesis of chronic disabling inflammatory diseases. FASEB J. 17, 2176-2183 (2003)

101. Straub, R. H. \& Schradin, C. Chronic inflammatory systemic diseases: an evolutionary trade-off between acutely beneficial but chronically harmful programs. Evol. Med. Public Health 2016, 37-51 (2016).

102. He, J. et al. Urinary sodium and potassium excretion and CKD progression. J. Am. Soc. Nephrol. 27, 1202-1212 (2015)

103. Mills, K. T. et al. Sodium excretion and the risk of cardiovascular disease in patients with chronic kidney disease. JAMA 315, 2200-2210 (2016)

104. Rodrīguez-Iturbe, B., Vaziri, N. D., Herrera-Acosta, J. $\&$ Johnson, R. J. Oxidative stress, renal infiltration of mmune cells, and salt-sensitive hypertension: all for one and one for all. Am. J. Physiol. Renal Physiol. 286, F606-F616 (2004).

105. Schatz, V. et al. Elementary immunology: $\mathrm{Na}^{+}$as a regulator of immunity. Pediatr. Nephrol. 32, 201-210 (2016)

106. Machnik, A. et al. Macrophages regulate saltdependent volume and blood pressure by a vascular endothelial growth factor-C-dependent buffering mechanism. Nat. Med. 15, 545-552 (2009).

107. Lee, N. K. An evolving integrative physiology: skeleton and energy metabolism. BMB Rep. 43, 579-583 (2010).

108. Amanzadeh, J. \& Reilly, R. F. Hypophosphatemia: an evidence-based approach to its clinical consequences and management. Nat. Clin. Pract. Nephrol. 2, 136-148 (2006).

109. Tonelli, M. et al. Relation between serum phosphate level and cardiovascular event rate in people with coronary disease. Circulation 112, 2627-2633 (2005).

110. Faul, C. et al. FGF23 induces left ventricular hypertrophy. J. Clin. Invest. 121, 4393-4408 (2011).

111. Ferron, M. \& Lacombe, J. Regulation of energy metabolism by the skeleton: osteocalcin and beyond. Arch. Biochem. Biophys. 561, 137-146 (2014).

112. Lee, N. K. et al. Endocrine regulation of energy metabolism by the skeleton. Cell 130, 456-469 (2007)

113. Rix, M., Andreassen, H., Eskildsen, P., Langdahl, B. \& Olgaard, K. Bone mineral density and biochemical markers of bone turnover in patients with predialysis chronic renal failure. Kidney Int. 56, 1084-1093 (1999).
114. Hinoi, E. et al. The sympathetic tone mediates leptin's inhibition of insulin secretion by modulating osteocalcin bioactivity. J. Cell Biol. 183, 1235-1242 (2008).

115. Hong, S.-H. et al. Changes in serum osteocalcin are not associated with changes in glucose or insulin for osteoporotic patients treated with bisphosphonate. J. Bone Metab. 20, 37-41 (2013).

116. Schwartz, A. V. et al. Effects of antiresorptive therapies on glucose metabolism: results from the FIT, HORIZON-PFT, and FREEDOM trials. J. Bone Miner. Res. 28, 1348-1354 (2013).

117. Vervloet, M. G. et al. Bone: a new endocrine organ at the heart of chronic kidney disease and mineral and bone disorders. Lancet Diabetes Endocrinol. 2, 427-436 (2014)

118. Holden, R. M. et al. Vitamins $\mathrm{K}$ and D status in stages 3-5 chronic kidney disease. Clin. J. Am. Soc. Nephrol. 5, 590-597 (2010)

119. McCabe, K. M. et al. Dietary vitamin K and therapeutic warfarin alter the susceptibility to vascula calcification in experimental chronic kidney disease. Kidney Int. 83, 835-844 (2013).

120. Okuno, S. et al. Significant inverse relationship between serum undercarboxylated osteocalcin and glycemic control in maintenance hemodialysis patients. Osteoporos. Int. 24, 605-612 (2013).

121. Patterson-Buckendahl, P. Osteocalcin is a stressresponsive neuropeptide. Endocr. Regul. 45, 99-110 (2011)

122. Kawai, M., Kinoshita, S., Shimba, S., Ozono, K. \& Michigami, T. Sympathetic activation induces skeletal Fgf23 expression in a circadian rhythm-dependent manner. J. Biol. Chem. 289, 1457-1466 (2014).

123. Grabner, A. et al. Activation of cardiac fibroblast growth factor receptor 4 causes left ventricular hypertrophy. Cell Metab. 22, 1020-1032 (2015)

124. Gutiérrez, O. M. et al. Fibroblast growth factor 23 and left ventricular hypertrophy in chronic kidney disease. Circulation 119, 2545-2552 (2009).

125. Dai, B. et al. A comparative transcriptome analysis identifying FGF23 regulated genes in the kidney of a mouse CKD model. PLOS ONE 7, e44161 (2012).

126. Wojcik, M. et al. The association of FGF23 levels in obese adolescents with insulin sensitivity. J. Pediatr. Endocrinol. Metab. 25, 687-690 (2012).

127. Mirza, M. A. I. et al. Circulating fibroblast growth factor- 23 is associated with fat mass and dyslipidemia in two independent cohorts of elderly individuals. Arterioscler. Thromb. Vasc. Biol. 31, 219-227 (2011)

128. Scialla, J. J. et al. Fibroblast growth factor- 23 and cardiovascular events in CKD. J. Am. Soc. Nephrol. 25, 349-360 (2014)

129. Xie, J., Yoon, J., An, S.-W., Kuro-o, M. \& Huang, C.-L. Soluble klotho protects against uremic cardiomyopathy independently of fibroblast growth factor 23 and phosphate. J. Am. Soc. Nephrol. 26, 1150-1160 (2015)

130. Fliser, D. et al. Fibroblast growth factor 23 (FGF23) predicts progression of chronic kidney disease: the Mild to Moderate Kidney Disease (MMKD) Study. J. Am. Soc. Nephrol. 18, 2600-2608 (2007).

131. Scialla, J. J. et al. Mineral metabolites and CKD progression in African Americans. J. Am. Soc. Nephrol. 24, 125-135 (2013)

132. Tripepi, G. et al. Competitive interaction between fibroblast growth factor 23 and asymmetric dimethylarginine in patients with CKD. J. Am. Soc Nephrol. 26, 1-10 (2014).

133. Sanz, A. B. et al. TWEAK and the progression of renal disease: clinical translation. Nephrol. Dial. Transplant. 29 (Suppl. 1), i54-i62 (2014).

134. David, V. et al. Inflammation and functional iron deficiency regulate fibroblast growth factor 23 production. Kidney Int. 89, 135-146 (2015)

135. Dounousi, E. et al. Intact FGF23 and aklotho during acute inflammation/sepsis in CKD patients. Eur. J. Clin. Invest. 46, 234-241 (2016)

136. Rossaint, J. et al. FGF23 signaling impairs neutrophil recruitment and host defense during CKD. 126 962-974 (2016)

137. Silswal, N. et al. FGF23 directly impairs endotheliumdependent vasorelaxation by increasing superoxide levels and reducing nitric oxide bioavailability. Am. J. Physiol. Endocrinol. Metab. 307, E426-E436 (2014)

138. Dounousi, E. et al. Effect of inflammation by acute sepsis on intact fibroblast growth factor 23 (iFGF23) and asymmetric dimethyl arginine (ADMA) in ckd patients. Nutr. Metab. Cardiovasc. Dis. 26, 80-83 (2015). 
139. Lopez, A. et al. Multiple-center, randomized, placebocontrolled, double-blind study of the nitric oxide synthase inhibitor 546C88: effect on survival in patients with septic shock. Crit. Care Med. 32, 21-30 (2004).

140. London, G. M., Marchais, S. J., Guérin, A. P. \& de Vernejoul, M.-C. Ankle-brachial index and bone turnover in patients on dialysis. J. Am. Soc. Nephrol. 26, 1-8 (2014).

141. Ng, K. W. Regulation of glucose metabolism and the skeleton. Clin. Endocrinol. (Oxf.) 75, 147-155 (2011).

142. Zoccali, C. et al. Leptin and biochemical markers of bone turnover in dialysis patients. J. Nephrol. 17, 253-260 (2004)

143. Grgurevic, L., Christensen, G. L., Schulz, T. J. \& Vukicevic, S. Bone morphogenetic proteins in inflammation, glucose homeostasis and adipose tissue energy metabolism. Cytokine Growth Factor Rev. 27, 105-118 (2016)

144. Luttropp, K. et al. Genotypic and phenotypic predictors of inflammation in patients with chronic kidney disease. Nephrol. Dial. Transplant. 31, 2033-2040 (2016).

145. Li, X., Yang, H.-Y. \& Giachelli, C. M. BMP-2 promotes phosphate uptake, phenotypic modulation, and calcification of human vascular smooth muscle cells. Atherosclerosis 199, 271-277 (2008).

146. Smith, E. R. et al. Phosphorylated fetuin-A-containing calciprotein particles are associated with aortic stiffness and a procalcific milieu in patients with pre-dialysis CKD. Nephrol. Dial. Transplant. 27, 1957-1966 (2012).

147. Muñoz-Félix, J. M., González-Núñez, M., MartínezSalgado, C. \& López-Novoa, J. M. TGF- $\beta /$ BMP proteins as therapeutic targets in renal fibrosis. Where have we arrived after 25 years of trials and tribulations? Pharmacol. Ther. 156, 44-58 (2015).

148. Massy, Z. A. \& Drueke, T. B. Activin receptor IIA ligand trap in chronic kidney disease: 1 drug to prevent 2 complications-or even more? Kidney Int. $\mathbf{8 9}$ 1180-1182 (2016)

149. Gruden, G., Landi, A. \& Bruno, G. Natriuretic peptides, heart, and adipose tissue: new findings and future developments for diabetes research. Diabetes Care 37, 2899-2908 (2014).

150. Engeli, S. et al. Natriuretic peptides enhance the oxidative capacity of human skeletal muscle. J. Clin. Invest. 122, 4675-4679 (2012).

151. Chiurchiū, V. et al. Brain natriuretic peptide (BNP) regulates the production of inflammatory mediators in human THP-1 macrophages. Regul. Pept. 148, 26-32 (2008).

152. Li, D. et al. Efficacy of B-type natriuretic peptide is coupled to phosphodiesterase $2 \mathrm{~A}$ in cardiac sympathetic neurons. Hypertension 66, 190-198 (2015).

153. Hagiwara, H. et al. Autocrine regulation of rat chondrocyte proliferation by natriuretic peptide $C$ and its receptor, natriuretic peptide receptor-B. J. Biol. Chem. 269, 10729-10733 (1994)

154. Suda, M. et al. C-type natriuretic peptide as an autocrine/paracrine regulator of osteoblast. Evidence for possible presence of bone natriuretic peptide system. Biochem. Biophys. Res. Commun. 223, 1-6 (1996).

155. Holliday, L. S., Dean, A. D., Greenwald, J. E. \& Glucks, S. L. C-type natriuretic peptide increases bone resorption in 1,25-dihydroxyvitamin D3-stimulated mouse bone marrow cultures. J. Biol. Chem. 270 18983-18989 (1995)

156. Suda, M. et al. Skeletal overgrowth in transgenic mice that overexpress brain natriuretic peptide. Proc. Natl Acad. Sci. USA 95, 2337-2342 (1998)

157. Zoccali, C. et al. Cardiac natriuretic peptides are related to left ventricular mass and function and predict mortality in dialysis patients. J. Am. Soc. Nephrol. 12, 1508-1515 (2001).

158. Gunta, S. S., Thadhani, R. I. \& Mak, R. H. The effect of vitamin D status on risk factors for cardiovascula disease. Nat. Rev. Nephrol. 9, 337-347 (2013).

159. Johal, M. \& Levin, A. Vitamin D and parathyroid hormone in general populations: understandings in 2009 and applications to chronic kidney disease. Clin. J. Am. Soc. Nephrol 4 1508-1514 (2009).

160. Koppe, L. et al. Insulin resistance in chronic kidney disease: new lessons from experimental models. Nephrol. Dial. Transplant. 29, 1666-1674 (2014).

161. Vanholder, R. \& Glorieux, G. The intestine and the kidneys: a bad marriage can be hazardous. Clin. Kidney J. 8, 168-179 (2015).
162. Wong, J. et al. Expansion of urease- and uricasecontaining, indole- and $\mathrm{p}$-cresol-forming and contraction of short-chain fatty acid-producing intestinal microbiota in ESRD. Am. J. Nephrol. 39 230-237 (2014).

163. Vaziri, N. D. et al. Chronic kidney disease alters intestinal microbial flora. Kidney Int. 83, 308-315 (2012).

164. Sumida, K. et al. Constipation and incident CKD J. Am. Soc. Nephrol. http://dx.doi.org/10.1681/ ASN. 2016060656 (2016).

165. Servillo, L., Giovane, A., Cautela, D., Castaldo, D. \& Balestrieri, M. L. The methylarginines NMMA, ADMA, and SDMA are ubiquitous constituents of the main vegetables of human nutrition. Nitric Oxide 30 , 43-48 (2013).

166. Koschinsky, T. et al. Orally absorbed reactive glycation products (glycotoxins): an environmental risk factor in diabetic nephropathy. Proc. Natl Acad. Sci. USA 94, 6474-6479 (1997)

167. Schepers, E. et al. Symmetric dimethylarginine as a proinflammatory agent in chronic kidney disease. Clin. J. Am. Soc. Nephrol. 6, 2374-2383 (2011).

168. Ravani, P. et al. Asymmetrical dimethylarginine predicts progression to dialysis and death in patients with chronic kidney disease: a competing risks modeling approach. J. Am. Soc. Nephrol. 16 2449-2455 (2005).

169. Zoccali, C. et al. Plasma concentration of asymmetrica dimethylarginine and mortality in patients with endstage renal disease: a prospective study. Lancet 358 2113-2117 (2001).

170. Tripepi, G. et al. Inflammation and asymmetric dimethylarginine for predicting death and cardiovascular events in ESRD patients. Clin. J. Am. Soc. Nephrol. 6, 1714-1721 (2011).

171. Baumann, M. Role of advanced glycation end products in hypertension and cardiovascular risk: human studies. J. Am. Soc. Hypertens. 6, 427-435 (2012).

172. Wikoff, W. R. et al. Metabolomics analysis reveals large effects of gut microflora on mammalian blood metabolites. Proc. Natl Acad. Sci. USA 106 3698-3703 (2009)

173. Poesen, R. et al. Renal clearance and intestinal generation of p-cresyl sulfate and indoxyl sulfate in CKD. Clin. J. Am. Soc. Nephrol. 8, 1508-1514 (2013).

174. Aronov, P. A. et al. Colonic contribution to uremic solutes. J. Am. Soc. Nephrol. 22, 1769-1776 (2011)

175. Tanaka, H. et al. p-cresyl sulfate induces osteoblast dysfunction through activating JNK and p38 MAPK pathways. Bone 56, 347-354 (2013).

176. Hirata, J. et al. Indoxyl sulfate exacerbates low bone turnover induced by parathyroidectomy in young adul rats. Bone 79, 252-258 (2015)

177. Pletinck, A. et al. Protein-bound uremic toxins stimulate crosstalk between leukocytes and vessel wall. J. Am. Soc. Nephrol. 24, 1981-1994 (2013)

178. Vanholder, R., Schepers, E., Pletinck, A., Nagler, E. V. $\&$ Glorieux, G. The uremic toxicity of indoxyl sulfate and p-cresyl sulfate: a systematic review. J. Am. Soc. Nephrol. 25, 1-11 (2014).

179. Piccoli, G. B. et al. Low protein diets in patients with chronic kidney disease: a bridge between mainstream and complementary-alternative medicines? BMC Nephrol. 17, 76 (2016).

180. Schulman, G. et al. Randomized placebo-controlled EPPIC trials of AST-120 in CKD. J. Am. Soc. Nephrol. 26, 1732-1746 (2015).

181. Cha, R.-H. et al. A randomized, controlled trial of oral intestinal sorbent AST-120 on renal function deterioration in patients with advanced renal dysfunction. Clin. J. Am. Soc. Nephrol. 11, 559-567 (2016)

182. Poesen, R. et al. Cardiovascular disease relates to intestinal uptake of $\mathrm{p}$-cresol in patients with chronic kidney disease. BMC Nephrol. 15, 87 (2014).

183. McCafferty, K. et al. Intestinal microbiota determine severity of myocardial infarction in rats. FASEB J. 26 1727-1735 (2012).

184. Wang, Z. et al. Gut flora metabolism of phosphatidylcholine promotes cardiovascular disease. Nature 472, 57-63 (2011)

185. Mueller, D. M. et al. Plasma levels of trimethylamine$\mathrm{N}$-oxide are confounded by impaired kidney function and poor metabolic control. Atherosclerosis 243 638-644 (2015)

186. Tang, W. H. W. et al. Gut microbiota-dependent trimethylamine $\mathrm{N}$-oxide (TMAO) pathway contributes to both development of renal insufficiency and mortality risk in chronic kidney disease. Circ. Res. 116 448-455 (2015).
187. Semba, R. D. et al. The association of serum choline with linear growth failure in young children from rural Malawi. Am. J. Clin. Nutr. 104, 191-197 (2016)

188. Galland, L. The gut microbiome and the brain. J. Med. Food 17, 1261-1272 (2014).

189. Brown, J. M. \& Hazen, S. L. The gut microbial endocrine organ: bacterially derived signals driving cardiometabolic diseases. Annu. Rev. Med. 66, 343-359 (2015).

190. Zhu, W. et al. Gut microbial metabolite TMAO enhances platelet hyperreactivity and thrombosis risk. Cell 165, 111-124 (2016).

191. Zgoda-Pols, J. R. et al. Metabolomics analysis reveals elevation of 3-indoxyl sulfate in plasma and brain during chemically-induced acute kidney injury in mice: investigation of nicotinic acid receptor agonists. Toxicol. Appl. Pharmacol. 255, 48-56 (2011).

192. Smith, P. A. The tantalizing links between gut microbes and the brain. Nature 526, 312-314 (2015)

193. Dou, L. et al. The cardiovascular effect of the uremic solute indole-3 acetic acid. J. Am. Soc. Nephrol. 26 876-887 (2015).

194. Shivanna, S. et al. The Aryl hydrocarbon receptor is a critical regulator of tissue factor stability and an antithrombotic target in uremia. J. Am. Soc. Nephrol. 27, 189-201 (2016).

195. Jankowski, J. et al. Increased plasma phenylacetic acid in patients with end-stage renal failure inhibits iNOS expression. J. Clin. Invest. 112, 256-264 (2003).

196. Rossi, M. et al. Synbiotics easing renal failure by improving gut microbiology (SYNERGY): a randomized trial. Clin. J. Am. Soc. Nephrol. 11, 1-9 (2016).

197. Garneata, L., Stancu, A., Dragomir, D., Stefan, G. \& Mircescu, G. Ketoanalogue-supplemented vegetarian very low-protein diet and CKD progression. J. Am. Soc Nephrol. 27, 2164-2176 (2016).

198. Simkhovich, B. Z., Kleinman, M. T. \& Kloner, R. A. Air pollution and cardiovascular injury. J. Am. Coll. Cardiol. 52, 719-726 (2008).

199. Sponholtz, T. R., Sandler, D. P., Parks, C. G. \& Applebaum, K. M. Occupational exposures and chronic kidney disease: possible associations with endotoxin and ultrafine particles. Am. J. Ind. Med. 59, 1-11 (2016).

200. Cutz, E. Hyperplasia of pulmonary neuroendocrine cells in infancy and childhood. Semin. Diagn. Pathol. 32, 420-437 (2015).

201. Whitsett, J. A. \& Morrisey, E. E. Inflammation. Modulating pulmonary inflammation. Science 351, 662-663 (2016)

202. Gu, X. et al. Chemosensory functions for pulmonary neuroendocrine cells. Am. J. Respir. Cell Mol. Biol. 50 637-646 (2014)

203. Kasacka, I. Quantitative characteristics of calcitoninproducing cells in the thyroid and lungs of uremic rats. Folia Histochem. Cytobiol. 46, 525-530 (2008).

204. Navaneethan, S. D. et al. Obstructive and restrictive lung function measures and CKD: National Health and Nutrition Examination Survey (NHANES) 2007-2012. Am. J. Kidney Dis. 68, 414-421 (2016).

205. Grassi, G. et al. Sympathetic nerve traffic and asymmetric dimethylarginine in chronic kidney disease. Clin. J. Am. Soc. Nephrol. 6, 2620-2627 (2011)

206. Hurtado, A., Escudero, E., Pando, J., Sharma, S. \& Johnson, R. J. Cardiovascular and renal effects of chronic exposure to high altitude. Nephrol. Dial. Transplant. 27 (Suppl. 4), iv11-iv16 (2012).

207. Mannino, D. M., Ford, E. S. \& Redd, S. C. Obstructive and restrictive lung disease and markers of inflammation: data from the Third National Health and Nutrition Examination. Am. J. Med. 114, 758-762 (2003)

208. Scheel, P. J., Liu, M. \& Rabb, H. Uremic lung: new insights into a forgotten condition. Kidney Int. 74 , 849-851 (2008).

209. Stenvinkel, P. \& Alvestrand, A. Inflammation in end stage renal disease: sources, consequences, and therapy. Semin. Dial. 15, 329-337 (2002).

210. Tripepi, G., Mallamaci, F. \& Zoccali, C. Inflammation markers, adhesion molecules, and all-cause and cardiovascular mortality in patients with ESRD: searching for the best risk marker by multivariate modeling. J. Am. Soc. Nephrol. 16 (Suppl. 1), S83-S88 (2005)

211. Zoccali, C. et al. Lung congestion as a risk factor in end-stage renal disease. Blood Purif. 36, 184-191 (2013).

212. Zoccali, C. et al. Pulmonary congestion predicts cardiac events and mortality in ESRD. J. Am. Soc Nephrol. 24, 639-646 (2013). 
213. Blanco, I., Piccari, L. \& Barberà, J. A. Pulmonary vasculature in COPD: the silent component Respirology 21, 984-994 (2016).

214. Bolignano, D. et al. High estimated pulmonary artery systolic pressure predicts adverse cardiovascular outcomes in stage 2-4 chronic kidney disease. Kidney Int. 88, 130-136 (2015).

215. Navaneethan, S. D. et al. Prevalence, predictors, and outcomes of pulmonary hypertension in CKD. J. Am. Soc. Nephrol. 27, 1-10 (2015).

216. Bolignano, D. et al. Pulmonary hypertension in CKD. Am. J. Kidney Dis. 61, 612-622 (2013).

217. Wang, J. et al. Impact of obstructive sleep apnea syndrome on endothelial function, arterial stiffening, and serum inflammatory markers: an updated metaanalysis and metaregression of 18 studies. J. Am. Heart Assoc. 4, e002454 (2015).

218. Abboud, F. \& Kumar, R. Obstructive sleep apnea and insight into mechanisms of sympathetic overactivity. J. Clin. Invest. 124, 1454-1457 (2014).

219. Kholdani, C., Fares, W. H. \& Mohsenin, V. Pulmonary hypertension in obstructive sleep apnea: is it clinically significant? A critical analysis of the association and pathophysiology. Pulm. Circ. 5, 220-227 (2015)

220. Ogna, A. et al. Sleep characteristics in early stages of chronic kidney disease in the HypnoLaus Cohort. Sleep 39, 945-953 (2015).

221. Sim, J. J., Rasgon, S. A. \& Derose, S. F. Review article managing sleep apnoea in kidney diseases. Nephrology (Carlton). 15, 146-152 (2010).

222. DiBona, G. F. Nervous kidney. Interaction between renal sympathetic nerves and the renin-angiotensin system in the control of renal function. Hypertension 36, 1083-1088 (2000)

223. Bourque, C. W., Oliet, S. H. \& Richard, D. Osmoreceptors, osmoreception, and osmoregulation. Front. Neuroendocrinol. 15, 231-274 (1994).

224. Stems, R. H. General principles of disorders of water balance (hyponatremia and hypernatremia) and sodium balance (hypovolemia and edema). UpToDate https://www.uptodate.com/contents/general-principlesof-disorders-of-water-balance-hyponatremia-andhypernatremia-and-sodium-balance-hypovolemia-andedema (2017).

225. Lechner, S. G. et al. The molecular and cellular identity of peripheral osmoreceptors. Neuron $69,332-344$ (2011).

226. Antunes-Rodrigues, J., de Castro, M., Elias, L. L., Valenca, M. M. \& McCann, S. M. Neuroendocrine control body fluid metabolism. Physiol. Rev. 84, 169-208 (2004)

227. Buijs, F. N. et al. The circadian system: a regulatory feedback network of periphery and brain. Physiology 31, 170-181 (2016)

228. Peek, C. B. et al. Circadian regulation of cellular physiology. Methods Enzymol. 552, 165-184 (2015).

229. Wuerzner, G., Firsov, D. \& Bonny, O. Circadian glomerular function: from physiology to molecular and therapeutical aspects. Nephrol. Dial. Transplant. 29 1475-1480 (2014).

230. Butterworth, R. F. in Basic Neurochemistry: Molecular, Cellular and Medical Aspects 6th edn (eds Siegel, G. J. et al.) (Lippincott-Raven, 1999).

231. Meyer, T. W. \& Hostetter, T. H. Approaches to uremia J. Am. Soc. Nephrol. 25, 2151-2158 (2014).

232. Kurella, M. et al. Chronic kidney disease and cognitive impairment in the elderly: the health, aging, and bod composition study. J. Am. Soc. Nephrol. 16, 2127-2133 (2005).

233. Seliger, S. L. Moderate renal impairment and risk of dementia among older adults: the Cardiovascula Health Cognition Study. J. Am. Soc. Nephrol. 15 1904-1911 (2004)

234. Chillon, J.-M., Massy, Z. A. \& Stengel, B. Neurological complications in chronic kidney disease patients. Nephrol. Dial. Transplant. 31, 1606-1614 (2015).

235. Sink, K. M. et al. Cerebral structural changes in diabetic kidney disease: African American-Diabetes Heart Study MIND. Diabetes Care 38, 206-212 (2015).

236. Degaspari, S. et al. Altered KLOTHO and NF-кB-TNF-o signaling are correlated with nephrectomy-induced cognitive impairment in rats. PLOS ONE 10 e0125271 (2015).

237. Smith, L. K. et al. $\beta 2$-microglobulin is a systemic pro-aging factor that impairs cognitive function and neurogenesis. Nat. Med. 21, 932-937 (2015).
238. Vanholder, R. et al. Review on uremic toxins: classification, concentration, and interindividual variability. Kidney Int. 63, 1934-1943 (2003).

239. Lee, Y. C. et al. Sleep apnea and the risk of chronic kidney disease: a nationwide population-based cohort study. Sleep 38, 213-221 (2015).

240. Molnar, M. Z. et al. Association of incident obstructive sleep apnoea with outcomes in a large cohort of US veterans. Thorax 70, 888-895 (2015)

241. Calvin, A. D., Albuquerque, F. N., Lopez-Jimenez, F. ¿ Somers, V. K. Obstructive sleep apnea, inflammation, and the metabolic syndrome. Metab. Syndr. Relat. Disord. 7, 271-278 (2009)

242. Zoccali, C. et al. Nocturnal hypoxemia, night-day arterial pressure changes and left ventricular geometry in dialysis patients. Kidney Int. $\mathbf{5 3}$, 1078-1084 (1998)

243. Daulatzai, M. A. Evidence of neurodegeneration in obstructive sleep apnea: relationship between obstructive sleep apnea and cognitive dysfunction in the elderly. J. Neurosci. Res. 93, 1778-1794 (2015)

244. Svatikova, A. et al. Serum amyloid a in obstructive sleep apnea. Circulation 108, 1451-1454 (2003).

245. Yaouhi, K. et al. A combined neuropsychological and brain imaging study of obstructive sleep apnea. J. Sleep Res. 18, 36-48 (2009)

246. Moodalbail, D. G. et al. Systematic review of structura and functional neuroimaging findings in children and adults with CKD. Clin. J. Am. Soc. Nephrol. 8 1429-1448 (2013)

247. Griva, K. et al. Cognitive impairment and 7-year mortality in dialysis patients. Am. J. Kidney Dis. 56 , 693-703 (2010).

248. Ahn, A. C., Tewari, M., Poon, C.-S. \& Phillips, R. S. The limits of reductionism in medicine: could systems biology offer an alternative? PLoS Med. 3, e208 (2006)

249. National Institutes of Health. What is the BRAIN Initiative? NIH http://www.braininitiative.nih.gov/ (2017)

250. Zawada, A. M. et al. Massive analysis of cDNA Ends (MACE) and miRNA expression profiling identifies proatherogenic pathways in chronic kidney disease. Epigenetics 9, 161-172 (2014).

251. Moreno, J. A. et al. The inflammatory cytokines TWEAK and TNFa reduce renal klotho expression through NFkB. J. Am. Soc. Nephrol. 22, 1315-1325 (2011)

252. Schepers, E. et al. Transcriptome analysis in patients with chronic kidney disease on hemodialysis disclosing a key role for CD16 + CX3CR1 + monocytes. PLoS ONE 10, e0121750 (2015).

253. Schiffer, E. et al. Markers of vascular disease in plasma from patients with chronic kidney disease identified by proteomic analysis. J. Hypertens. $\mathbf{2 9}$ 783-790 (2011).

254. Gajjala, P. R., Fliser, D., Speer, T., Jankowski, V. \& Jankowski, J. Emerging role of post-translational modifications in chronic kidney disease and cardiovascular disease. Nephrol. Dial. Transplant. 30, 1814-1824 (2015)

255. Toyohara, T. et al. Metabolomic profiling of uremic solutes in CKD patients. Hypertens. Res. 33. 944-952 (2010)

256. Kalim, S. et al. A plasma long-chain acylcarnitine predicts cardiovascular mortality in incident dialysis patients. J. Am. Heart Assoc. 2, e000542 (2013).

257. Posada-Ayala, M. et al. Identification of a urine metabolomic signature in patients with advancedstage chronic kidney disease. Kidney Int. 85, 103-111 (2014).

258. Koeth, R. A. et al. Intestinal microbiota metabolism of L-carnitine, a nutrient in red meat, promotes atherosclerosis. Nat. Med. 19, 576-585 (2013).

259. Bain, M. A., Faull, R., Fornasini, G., Milne, R. W. $\delta$ Evans, A. M. Accumulation of trimethylamine and trimethylamine- $\mathrm{N}$-oxide in end-stage renal disease patients undergoing haemodialysis. Nephrol. Dial. Transplant. 21, 1300-1304 (2006)

260. Duranton, F. et al. Normal and pathologic concentrations of uremic toxins. J. Am. Soc. Nephrol. 23, 1258-1270 (2012).

261. Cisek, K., Krochmal, M., Klein, J. \& Mischak, H. The application of multi-omics and systems biology to identify therapeutic targets in chronic kidney disease. Nephrol. Dial. Transplant. 31, 2003-201 (2015)
262. Green, E. D. et al. Charting a course for genomic medicine from base pairs to bedside. Nature $\mathbf{4 7 0}$ 204-213 (2011)

263. Wanichthanarak, K., Fahrmann, J. F. \& Grapov, D. Genomic, proteomic, and metabolomic data integration strategies. Biomark. Insights 10 (Suppl. 4), 1-6 (2015).

264. McMullan, D. What is personalized medicine? GenomeMag http://genomemag.com/what-ispersonalized-medicine/\# (2016).

265. de Zeeuw, D. et al. Bardoxolone methyl in type 2 diabetes and stage 4 chronic kidney disease. N. Engl. J. Med. 369, 2492-2503 (2013)

266. Guo, S., Zou, J. \& Wang, G. Advances in the proteomic discovery of novel therapeutic targets in cancer. Drug Des. Devel. Ther. 7, 1259-1271 (2013).

267. Merchant, M. L. Mass spectrometry in chronic kidney disease research. Adv. Chron. Kidney Dis. 17 455-468 (2010).

268. Papadopoulos, T. et al. Omics databases on kidney disease: where they can be found and how to benefit from them. Clin. Kidney J. 9, 343-352 (2016).

269. Zoccali, C., Tripepi, G., Dounousi, E. \& Mallamaci, F. Chronic kidney disease (CKD) as a systemic disease: whole body autoregulation and inter-organ crosstalk. Kidney Blood Press. Res. 39, 134-141 (2014).

270. Vanholder, R. et al. Clinical management of the uraemic syndrome in chronic kidney disease. lancet. Diabetes Endocrinol. 4, 360-373 (2016).

271. Dhabhar, F. S., Miller, A. H., McEwen, B. S. \& Spencer, R. L. Stress-induced changes in blood leukocyte distribution. Role of adrenal steroid hormones. J. Immunol. 157, 1638-1644 (1996).

272. Kohm, A. P. \& Sanders, V. M. Norepinephrine and beta 2-adrenergic receptor stimulation regulate $C D 4+T$ and $\mathrm{B}$ lymphocyte function in vitro and in vivo. Pharmacol. Rev 53, 487-525 (2001).

273. Lieberman, H. R., Garfield, G., Waldhauser, F., Lynch, H. J. \& Wurtman, R. J. Possible behavioral consequences of light-induced changes in melatonin availability. Ann. NY Acad. Sci. 453, 242-252 (1985).

274. Sassin, J. F. et al. Human growth hormone release: relation to slow-wave sleep and sleep-walking cycles. Science 165, 513-515 (1969).

275. Sassin, J. F., Frantz, A. G., Weitzman, E. D. \& Kapen, S. Human prolactin: 24-hour pattern with increased release during sleep. Science 177 1205-1207 (1972).

276. Cernysiov, V., Gerasimcik, N., Mauricas, M. $\delta$ Girkontaite, I. Regulation of T-cell-independent and T-cell-dependent antibody production by circadian rhythm and melatonin. Int. Immunol. 22, 25-34 (2010).

277. Dimitrov, S., Lange, T., Fehm, H. L. \& Born, J. A regulatory role of prolactin, growth hormone, and corticosteroids for human T-cell production of cytokines. Brain. Behav. Immun. 18, 368-374 (2004).

278. Ericsson, A., Arias, C. \& Sawchenko, P. E. Evidence for an intramedullary prostaglandin-dependent mechanism in the activation of stress-related neuroendocrine circuitry by intravenous interleukin- 1 . J. Neurosci. 17, 7166-7179 (1997).

279. Cutolo, M. et al. Circadian rhythms: glucocorticoids and arthritis. Ann. NY Acad. Sci. 1069, 289-299 (2006).

280. Semenza, G. L. Hypoxia-inducible factor 1 and cardiovascular disease. Annu. Rev. Physiol. 76, 39-56 (2014)

281. Thangarajah, H. et al. HIF-1 alpha dysfunction in diabetes. Cell Cycle 9, 75-79 (2010).

282. Zhu, X.-Y. et al. Cortical microvascular remodeling in the stenotic kidney: role of increased oxidative stress. Arterioscler. Thromb. Vasc. Biol. 24, 1854-1859 (2004).

\section{Author contributions}

All authors researched the data, discussed the article's content, wrote the text and reviewed or edited the article before submission.

\section{Competing interests statement}

The authors declare no competing interests.

\section{Publisher's note}

Springer Nature remains neutral with regard to jurisdictional claims in published maps and institutional affiliations. 
CORRIGENDUM

\section{The systemic nature of CKD}

Carmine Zoccali, Raymond Vanholder, Ziad A. Massy, Alberto Ortiz, Pantelis Sarafidis, Friedo W. Dekker, Danilo Fliser, Denis Fouque, Gunnar H. Heine, Kitty J. Jager, Mehmet Kanbay, Francesca Mallamaci,

Gianfranco Parati, Patrick Rossignol, Andrzej Wiecek, Gerard London \& on behalf of the European Renal and Cardiovascular Medicine (EURECA-m) Working Group of the European Renal Association - European Dialysis Transplantation Association (ERA-EDTA)

Nature Reviews Nephrology 13, 344-358 (2017)

In the html and pdf versions of this article originally published online the permissions line for Figure 1 was not included. This error has now been corrected online and in print. 